University of Wollongong

Research Online

Faculty of Engineering and Information

Faculty of Engineering and Information

Sciences - Papers: Part A

Sciences

$1-1-2014$

(Strong) multidesignated verifiers signatures secure against rogue key attack

Man Ho Au

University of Wollongong, aau@uow.edu.au

Guomin Yang

University of Wollongong, gyang@uow.edu.au

Willy Susilo

University of Wollongong, wsusilo@uow.edu.au

Yunmei Zhang

University of Wollongong, yz841@uowmail.edu.au

Follow this and additional works at: https://ro.uow.edu.au/eispapers

Part of the Engineering Commons, and the Science and Technology Studies Commons

Research Online is the open access institutional repository for the University of Wollongong. For further information contact the UOW Library: research-pubs@uow.edu.au 


\title{
(Strong) multidesignated verifiers signatures secure against rogue key attack
}

\begin{abstract}
Designated verifier signatures (DVS) allow a signer to create a signature whose validity can only be verified by a specific entity chosen by the signer. In addition, the chosen entity, known as the designated verifier, cannot convince any body that the signature is created by the signer. Multidesignated verifiers signatures (MDVS) are a natural extension of DVS in which the signer can choose multiple designated verifiers. DVS and MDVS are useful primitives in electronic voting and contract signing. In this paper, we investigate various aspects of MDVS and make two contributions. Firstly, we revisit the notion of unforgeability under rogue key attack on MDVS. In this attack scenario, a malicious designated verifier tries to forge a signature that passes through the verification of another honest designated verifier. A common counter-measure involves making the knowledge of secret key assumption in which an adversary is required to produce a proof-of-knowledge of the secret key. We strengthened the existing security model to capture this attack and propose a new construction that does not rely on the knowledge of secret key assumption. Secondly, we propose a generic construction of strong MDVS.
\end{abstract}

\section{Keywords}

verifiers, signatures, secure, multidesignated, against, strong, rogue, key, attack

Disciplines

Engineering | Science and Technology Studies

\section{Publication Details}

Au, M. Ho., Yang, G., Susilo, W. \& Zhang, Y. (2014). (Strong) multidesignated verifiers signatures secure against rogue key attack. Concurrency and Computation: Practice and Experience, 26 (8), 1574-1592. 


\title{
(Strong) Multi-Designated Verifiers Signatures Secure Against Rogue Key Attack
}

\author{
Man Ho Au*, Guomin Yang, Willy Susilo and Yunmei Zhang \\ Centre for Computer and Information Security Research(CCISR), School of Computer Science and Software \\ Engineering, University of Wollongong, Australia
}

\begin{abstract}
SUMMARY
Designated verifier signatures (DVS) allow a signer to create a signature whose validity can only be verified by a specific entity chosen by the signer. In addition, the chosen entity, known as the designated verifier, cannot convince any body that the signature is created by the signer. Multi-designated verifiers signatures (MDVS) are a natural extension of DVS in which the signer can choose multiple designated verifiers. DVS and MDVS are useful primitives in electronic voting and contract signing. In this paper, we investigate various aspects of MDVS and make two contributions. Firstly, we revisit the notion of unforgeability under rogue key attack on MDVS. In this attack scenario, a malicious designated verifier tries to forge a signature that passes through the verification of another honest designated verifier. A common counter-measure involves making the knowledge of secret key assumption (KOSK) in which an adversary is required to produce a proof-of-knowledge of the secret key. We strengthened the existing security model to capture this attack and propose a new construction that does not rely on the KOSK assumption. Secondly, we propose a generic construction of strong MDVS. Copyright (c) 2013 John Wiley \& Sons, Ltd.
\end{abstract}

Received ...

KEY WORDS: Designated Verifier Signature, Rogue Key Attack, Public Key Cryptography

\section{INTRODUCTION}

Designated verifier signatures/proofs (DVS/DVP) were introduced by Jakobsson, Sako and Impagliazzo [1], and independently by Chaum [2] in 1996. A DVS scheme allows a signer Alice to convince a designated verifier Bob that Alice has endorsed the message while Bob cannot transfer this conviction to anyone else. The underlying principle of DVS is that a signature is a non-interactive proof that asserts the validity of the statement "Alice has endorsed a message" or "the signer has Bob's secret key". While Bob is convinced that Alice has endorsed the message, he cannot convince Carol as the proof could have been produced by Bob himself. In the same paper, Jakobsson et al. introduce the concept of strong DVS (SDVS) in which the private key of Bob is required to verify the signature. Recall that DVS itself discloses the information that the signature is produced by Alice or Bob. If an external party, Carol, is confident that Bob has not created the signature, she knows Alice has endorsed the message. An example is that the signature is captured by Carol before it reaches Bob. This requirement is formalized as privacy of signer's identity in [3]. It is required that without Bob's private key, Carol cannot tell if a signature is created by Alice or another signer.

In [1], the concept of multiple verifiers has been discussed and in the rump session of Crypto'03, Desmedt [4] proposed the notion of multi-designated verifiers signatures (MDVS)

\footnotetext{
${ }^{*}$ Correspondence to: School of Computer Science and Software Engineering, University of Wollongong, NSW 2522, Australia. Email: aau@uow.edu.au 
as a generalization of DVS. It was later formalized in [5]. Since then, a number of MDVS constructions $[1,5,6,7,8,9,10,11,12,13,14,15]$ with different features in different settings have been proposed. Interested readers may refer to [15] for a survey.

The problem of rogue key attack in DVS was first discussed in [1]. In the discussion, the goal of a malicious verifier Bob is to convince an external party Carol that the signer Alice has endorsed the message. For example, Bob can create his public key as the output of a hash function using a random number as input. Later, when Bob reveals the value of the random number, everyone will be convinced that the signatures must have been created by Alice. One of the counter-measures suggested is to require Bob to prove the knowledge of his secret key. Another type of rogue key attack specifically targeting MDVS was discussed in [16]. In this attack, a malicious verifier Carol creates her public key as a function of other honest verifiers' public keys so that she could create a signature that passes the verification of other honest verifiers. Again, the suggested counter-measure is to require the verifier to prove the knowledge of her secret key. Note that no formal model has been proposed to capture the attack. We remark that the two types of rogue key attacks are different in nature. The former is against non-transferability while the latter is against unforgeability. In this paper, our focus is on the latter.

As discussed, a counter-measure against rogue key attack in MDVS is to require the adversary to produce a proof-of-knowledge of the secret key. In practice, this implies all users would have to produce a proof-of-knowledge of the secret key to the certification authority (CA) before the CA certifies the corresponding public key. This solution requires a change in the current PKI and is regarded as costly [17]. Thus, it is desirable to design MDVS secure against rogue key attack in the plain model. In respond to this, we provide a partial solution by proposing the first MDVS scheme that is formally proven unforgeable under rogue key attack.

It is known that if we encrypt the DVS under the designated verifier's public key, the resulting scheme would be a strong DVS. Nonetheless, a subtle issue discussed in [5] prevent such generic transformation to be applicable to the case of MDVS. Specifically, the challenge is to ensure correctness of the resulting scheme since it is entirely possible for a signer to encrypt different values under different designated verifier's public key so that a signature could be regarded as valid by some of the designated verifiers only. We tackle this issue with an hybrid encryption using a simple oneway secure encryption and a symmetric encryption and show that the unforgeability under rogue key attack is preserved in our generic transformation. Specifically, we make the following contributions.

\subsection{Contribution.}

1. We present a formal definition for MDVS that captures existential forgery under rogue key attack.

2. We propose a construction that is provably secure against rogue key attack in the random oracle model.

3. We present two generic constructions of strong MDVS (with concrete instantiations) secure against rogue key attack in the random oracle model.

1.1.1. Organization. The rest of the paper is organized as follows. In Section 2, we review the syntax of a MDVS scheme and its security definitions. We discuss the rogue key attack on MDVS, its formal definition and our proposed solution in Section 3. In Section 4 and 5, we present generic constructions of strong MDVS together with concrete instantiations of the building blocks. We conclude our paper in Section 6.

\section{PRELIMINARY}

If $n$ is a positive integer, we use $[n]$ to denote the set $\{1, \ldots, n\}$. We review the following well-known computational assumptions.

Definition 1 (DL Assumption)

Let $\mathbb{G}=\langle g\rangle$ be a cyclic group of prime order $p$. The discrete logarithm assumption states that 
given a tuple $(g, Z) \in(\mathbb{G}, \mathbb{G})$, it is computationally infeasible to compute the value $z \in \mathbb{Z}_{p}$ such that $Z=g^{z}$.

Definition 2 (CDH Assumption)

Let $\mathbb{G}=\langle g\rangle$ be a cyclic group of prime order $p$. The computational Diffie-Hellman assumption states that given a tuple $\left(g, g^{a}, g^{b}\right) \in(\mathbb{G}, \mathbb{G}, \mathbb{G})$, it is computationally infeasible to compute the value $g^{a b}$.

We also review a variant of the $\mathrm{CDH}$ assumption, the divisible computation Diie-Hellman assumption (DCDH). It has been shown in [18] that the DCDH assumption and the $\mathrm{CDH}$ assumption are equivalent.

Definition 3 (DCDH Assumption)

Let $\mathbb{G}=\langle g\rangle$ be a cyclic group of prime order $p$. The divisible computational Diffie-Hellman assumption states that given a tuple $\left(g, g^{a}, g^{b}\right) \in(\mathbb{G}, \mathbb{G}, \mathbb{G})$, it is computationally infeasible to compute the value $g^{a / b}$.

\subsection{Syntax}

We adapt the definitions and security models of MDVS from various literatures [5, 9]. A MDVS scheme consists of four algorithms, namely, Setup, Gen, Sign, Verify, whose functions are enumerated below.

param $\leftarrow \operatorname{Setup}\left(1^{\lambda}\right)$ : On input a security parameter $\lambda$, this algorithm outputs the public parameter param for the system. Note that this algorithm is optional if all users could generate their key pairs without any coordination. Nonetheless, to the best of our knowledge, all existing schemes requires the users to create their keys based on some commonly known system parameters. We assume param is an implicit input to all algorithms listed below.

$(\mathrm{pk}, \mathrm{sk}) \leftarrow \mathrm{Gen}()$ : This algorithm outputs a key pair (pk, sk) for a user (who can take the role of a signer or a designated verifier). If ( $\mathrm{pk}, \mathrm{sk}$ ) is an output of the algorithm Gen(), we say pk is the corresponding public key of sk (and vice versa).

$(\sigma, \mathcal{V}) \leftarrow \operatorname{Sign}\left(\mathrm{sk}_{S}, \mathcal{V}, m\right)$ : On input a message $m$, a secret key of a signer $\mathrm{sk}_{S}$ (whose public key is $\mathrm{pk}_{S}$ ) and a set of designated verifiers' public keys $\mathcal{V}$, this algorithm outputs a signature $\sigma$, which is a designated verifier signature of $m$ with respect to the public key $\mathrm{pk}_{S}$.

valid/invalid $\leftarrow \operatorname{Verify}\left(\mathrm{pk}_{S}, \sigma, \mathcal{V}, m, \mathrm{sk}_{V}\right):$ On input a public key $\mathrm{pk}_{S}$, a message $m$, a signature $\sigma$ with a set of designated verifiers' public keys $\mathcal{V}$ and a private key $\mathrm{sk}_{V}$ such that the corresponding public key $\mathrm{pk}_{V} \in \mathcal{V}$, this algorithm verifies the signature and outputs valid/invalid.

A MDVS scheme must possess Correctness, Unforgeability and Source-Hiding, to be reviewed below.

Correctness. For any security parameter $\lambda$ and param $\leftarrow \operatorname{Setup}\left(1^{\lambda}\right),\left(\mathrm{pk}_{S}, \mathrm{sk}_{S}\right) \leftarrow \operatorname{Gen}()$ and $\mathcal{V}=\left\{\mathrm{pk}_{V_{1}}, \ldots, \mathrm{pk}_{V_{n}}\right\}$ such that $\left(\mathrm{pk}_{V_{i}}, \mathrm{sk}_{V_{i}}\right) \leftarrow \operatorname{Gen}()$ for $i \in[n]$. For any message $m$, if $(\sigma, \mathcal{V}) \leftarrow$ $\operatorname{Sign}\left(\mathrm{sk}_{S}, \mathcal{V}, m\right)$, then valid $\leftarrow \operatorname{Verify}\left(\mathrm{pk}_{S}, \sigma, \mathcal{V}, m, \mathrm{sk}_{V_{i}}\right)$ for all $i \in[n]$. Furthermore, for any values $\sigma, \mathcal{V}, m, \mathrm{pk}_{S}$, if there exists a private key $\mathrm{sk}_{V}$ such that its corresponding public key $\mathrm{pk}_{V} \in \mathcal{V}$ and that valid $\leftarrow \operatorname{Verify}\left(\mathrm{pk}_{S}, \sigma, \mathcal{V}, m, \mathrm{sk}_{V}\right)$, then for any private key $\mathrm{sk}_{V^{\prime}}$, it holds that valid $\leftarrow \operatorname{Verify}\left(\mathrm{pk}_{S}, \sigma, \mathcal{V}, m, \mathrm{sk}_{V^{\prime}}\right)$ if the corresponding public key $\mathrm{pk}_{V^{\prime}} \in \mathcal{V}$.

Unforgeability. The following game between a challenger $\mathcal{C}$ and an adversary $\mathcal{A}$ formally captures the requirement of Unforgeability.

Setup $\mathcal{C}$ invokes Setup $\left(1^{\lambda}\right)$ and subsequently $\operatorname{Gen}()$ to obtain (param, ( $\mathrm{pk}_{S}, \mathrm{sk}_{S}$ ), $\left.\left\{\left(\mathrm{pk}_{V_{i}}, \mathrm{sk}_{V_{i}}\right)\right\}_{i \in[n]}\right)$. Denote the set $\left\{\mathrm{pk}_{V_{i}}\right\}_{i \in[n]}$ by $\mathcal{V}$. (param, $\left.\mathrm{pk}_{S}, \mathcal{V}\right)$ is given to $\mathcal{A}$.

Query $\mathcal{A}$ is allowed to make the following queries: 
- Corruption Query. $\mathcal{A}$ submits a public key $\mathrm{pk}_{V} \in \mathcal{V}$ and receives $\mathrm{sk}_{V}$.

- Signature Query. $\mathcal{A}$ submits a message $m$ and receives $(\sigma, \mathcal{V}) \leftarrow \operatorname{Sign}\left(\mathrm{sk}_{S}, \mathcal{V}, m\right)$.

Output $\mathcal{A}$ submits $\left(\sigma^{*}, m^{*}\right)$ and wins if and only if

1. There exists a public key $\mathrm{pk}_{V^{*}} \in \mathcal{V}$ such that valid $\leftarrow \operatorname{Verify}\left(\mathrm{pk}_{S}, \sigma^{*}, \mathcal{V}, m^{*}, \mathrm{sk}_{V^{*}}\right)$.

2. $\mathcal{A}$ has not submitted a Signature Query with input $m^{*}$.

3. There exists a public key $\mathrm{pk}_{V} \in \mathcal{V}$ such that $\mathcal{A}$ has not submitted a Corruption Query as input.

Definition 4 (Unforgeability)

A MDVS scheme is unforgeable if no PPT adversary wins the above game with non-negligible probability.

As stated in [5], the adversary is not given an oracle for signature verification as he can verify any signatures by corrupting some of the verifiers.

Source hiding. It means that given a message $m$ and a signature $(\sigma, \mathcal{V})$, it is infeasible to determine who from the original signer or the designated verifiers all together created the signature, even if all the secret keys are known. The formal definition is adapted from Definition 3 of [19] for normal DVS into that for MDVS.

Definition 5 (Source Hiding)

A MDVS scheme is source hiding if there exists a PPT simulation algorithm Sim that on input a public key $\mathrm{pk}_{S}$, a set of key pairs $\left(\mathrm{pk}_{V_{i}}, \mathrm{sk}_{V_{i}}\right)_{i \in[n]}$ and a message $m$, outputs a tuple $(\sigma, \mathcal{V})$ (such that $\mathcal{V}=\left\{\mathrm{pk}_{V_{i}}\right\}_{i \in[n]}$ ) that is indistinguishale to $(\sigma, \mathcal{V}) \leftarrow \operatorname{Sign}\left(\mathrm{sk}_{S}, \mathcal{V}, m\right)$ (where $\mathrm{sk}_{S}$ is the corresponding private key of $\mathrm{pk}_{S}$ ). In other words, for all PPT algorithmn $\mathcal{D}$, for any security parameter $\lambda$, param $\leftarrow \operatorname{Setup}\left(1^{\lambda}\right),\left(\mathrm{pk}_{S}, \mathrm{sk}_{S}\right) \leftarrow \operatorname{Gen}(),\left\{\left(\mathrm{pk}_{V_{i}}, \mathrm{sk}_{V_{i}}\right) \leftarrow \operatorname{Gen}()\right\}_{i \in[n]}$ and any message $m$, it holds that:

$$
\operatorname{Pr}\left[\begin{array}{c}
\left(\sigma_{0}, \mathcal{V}\right) \leftarrow \operatorname{Sign}\left(\mathrm{sk}_{S}, \mathcal{V}, m\right) \\
\left(\sigma_{1}, \mathcal{V}\right) \leftarrow \operatorname{Sim}\left(\mathrm{pk}_{S},\left\{\mathrm{pk}_{V_{i}}, \mathrm{sk}_{V_{i}}\right\}_{i \in[n]}, m\right) \\
b \in_{R}\{0,1\} \\
b^{\prime} \leftarrow \mathcal{D}\left(\sigma_{b}, \mathrm{pk}_{S}, \mathrm{sk}_{S},\left\{\mathrm{pk}_{V_{i}}, \mathrm{sk}_{V_{i}}\right\}_{i \in[n]}, m\right)
\end{array}: b=b^{\prime}\right]-1 / 2 \mid=\operatorname{neg}(\lambda)
$$

where $\operatorname{negl}(\lambda)$ represents a negligible function in $\lambda$. A function negl $(\lambda)$ is said to be negligible in $\lambda$ if for all polynomial $q(\cdot)$, there exists a value $k_{0}$ such that for every $\lambda>k_{0}$, negl $(\lambda)<1 / q(\lambda)$.

\subsection{Strong Multi-Designated Verifiers Signatures}

Strong Multi-Designated Verifiers Signatures. It is desirable in many scenarios that, besides the signer and the verifier, a third party cannot tell if a signature for the verifier is created by that particular signer or by someone else. This concept appeared in [1] and is formally defined as privacy of signer's identity (PSI) in [20]. This applies to the case of multiple designated verifiers and the property PSI for MDVS is defined in [5].

Privacy of signer's identity. The following game between a challenger $\mathcal{C}$ and an adversary $\mathcal{A}$ formally captures the requirement of $P S I$.

Setup $\mathcal{C}$ invokes Setup $\left(1^{\lambda}\right)$ and subsequently Gen () to obtain (param, $\left(\mathrm{pk}_{S_{0}}, \mathrm{sk}_{S_{0}}\right),\left(\mathrm{pk}_{S_{1}}, \mathrm{sk}_{S_{1}}\right)$, $\left.\left\{\left(\mathrm{pk}_{V_{i}}, \mathrm{sk}_{V_{i}}\right)\right\}_{i \in[n]}\right)$. Denote the set $\left\{\mathrm{pk}_{V_{i}}\right\}_{i \in[n]}$ by $\mathcal{V}$. (param, $\left.\mathrm{pk}_{S_{0}}, \mathrm{pk}_{S_{1}}, \mathcal{V}\right)$ is given to $\mathcal{A}$.

Query $\mathcal{A}$ is allowed to make the following queries:

- Verification Query. $\mathcal{A}$ submits $\left(m, \sigma, \mathcal{V}, \mathrm{pk}_{S_{c}}: c \in\{0,1\}, V \in \mathcal{V}\right)$ and receives valid/invalid $\leftarrow \operatorname{Verify}\left(\mathrm{pk}_{S_{c}}, \sigma, \mathcal{V} \cup \mathrm{pk}_{V}, m, \mathrm{sk}_{V}\right)$

- Signature Query. $\mathcal{A}$ submits a message $m$, a bit $b$ and receives $(\sigma, \mathcal{V}) \leftarrow \operatorname{Sign}\left(\operatorname{sk}_{S_{b}}, \mathcal{V}\right.$, $m)$. 
Challenge At some point $\mathcal{A}$ submits a message $m^{*} . \mathcal{C}$ flips a fair coin $b$ and returns $\left(\sigma^{*}, \mathcal{V}\right) \leftarrow$ $\operatorname{Sign}\left(\mathrm{sk}_{S_{b}}, \mathcal{V}, m\right)$.

Query $\mathcal{A}$ continues to make verification and signature queries.

Output $\mathcal{A}$ submits a bit $b^{\prime}$ and wins if and only if $b^{\prime}=b$.

$\mathcal{A}$ 's advantage in the game PSI is defined as the probability that $\mathcal{A}$ wins the game minus $1 / 2$.

Definition 6 (Privacy of signer's identity)

A MDVS scheme is said to possess privacy of signer's identity if no PPT adversary has nonnegligible advantage in game PSI.

A strong MDVS scheme is a MDVS scheme that possesses privacy of signer's identity.

\section{ROUGE KEY ATTACK IN MDVS AND ITS SOLUTION}

We first review the generic construction of MDVS from discrete logarithm-based ring signatures [5]. In the next subsection, we describe how a malicious designated verifier could launch a rogue key attack to make an honest verifier into accepting a forged signature. We stress that this attack is outside the original security model and does not imply the scheme is insecure. Rather, we would like to show that a signature that passes the verification of a particular honest designated verifier could have been created by a real signer or some other malicious verifiers. Finally, we propose a fix.

\subsection{Generic Construction of MDVS [5]}

The generic construction utilizes ring signatures as building blocks and requires that all the keys are discrete logarithm-based. Readers are referred to [21] for the formal definition of a ring signature scheme. Roughly speaking, a ring signature is a signature created from one of the possible signers in a set of signers (often called a ring of signers). The ring of signers are created in an ad-hoc manner by the actual signer. The formation is spontaneous in that the members can be completely unaware of being conscripted into the ring. In the generic construction of MDVS, ring signatures supporting a ring size of 2 is required.

- Setup. This is equivalent to the parameter generation of the ring signature scheme (if any).

- Gen. This is equivalent to the key generation of the ring signature scheme. The generic construction requires the key of the ring signature to be of the form $\left(g^{x}, x\right)$ where $g$ is included in the parameter, $x$ is the signing key and $g^{x}$ is the corresponding public key.

- Sign. Let the signer's key pair be $\left(g^{x_{S}}, x_{S}\right)$ and the set of designated verifiers' key pairs be $\left\{\left(g^{x_{V_{i}}}, x_{V_{i}}\right)\right\}$ for $i=1$ to $n$. The signer computes $g^{X_{V}}=\prod_{i \in[n]} g^{x_{V_{i}}}$. Next, the signer creates a ring signature on message $m$ on the ring $\left\{g^{x_{S}}, g^{x_{V}}\right\}$ using the secret key $x_{S}$. Denote the output as $\sigma$. This value, together with the set $\left\{g^{x_{V_{i}}}\right\}_{i \in[n]}$, is outputted as the multi-designated verifier signature.

- Verify. To verify the signature $\left(\sigma,\left\{g^{x_{V_{i}}}\right\}_{i \in[n]}\right)$ on message $m$, a verifier computes $g^{X_{V}}=$ $\prod_{i \in[n]} g^{x_{V_{i}}}$. Then it employs the verification algorithm of the ring signature scheme on the ring $\left\{g^{X_{S}}, g^{X_{V}}\right\}$.

The unforgeability property comes from the fact that to create a ring signature on the ring $\left\{g^{x_{S}}, g^{x_{V}}\right\}$, one needs to know $x_{S}$ or $x_{V}$. Since the adversary does not know $x_{S}$ or $x_{V}^{\dagger}$, forging a signature implies breaking the unforgeability of the underlying ring signature scheme. On the other hand, the source hiding property comes from the fact that if all secret keys of the verifiers are known, one can construct a PPT Sim which computes $x_{V}=\sum_{i \in[n]} x_{V_{i}}$ and uses it to create a ring signature on behalf of the ring $\left\{g^{x_{S}}, g^{x_{V}}\right\}$. Due to the anonymity of ring signature, no PPT algorithm can distinguish a signature created by the real signer using $x_{S}$ or by Sim using $x_{V}$.

\footnotetext{
${ }^{\dagger}$ Since the adversary cannot corrupt all the verifiers, it does not know the value $x_{V}$, which is equal to $\sum_{i \in[n]} x_{V_{i}}$.
} 


\subsection{Rouge Key Attack and Its Defence}

Existential Forgery under Rouge Key Attack. Rouge key attack against a concrete scheme in [5] has been discussed in [16]. Here we extend the attack to the generic construction of [5]. Suppose an adversary's goal is to convince an honest designated verifier into accepting a forged signature. Let $g^{x_{S}}, g^{x_{V^{\prime}}}$ be the public keys of the targeted signer and designated verifier respectively. To cheat the verifier, the adversary randomly generates a value $x_{A}$ and crafts a mal-formed public key $K=g^{x_{A}} / g^{x_{V^{\prime}}}$. Next, the adversary computes $g^{x_{V}}=K g^{x_{V^{\prime}}}=g^{x_{A}}$. Since the adversary is in possession of $x_{A}$, he can create a ring signature on the ring $\left\{g^{x_{S}}, g^{x_{V}}\right\}$. He outputs the signature, together with the set of designated verifiers as $\left\{g^{x_{V^{\prime}}}, K\right\}$. Consequently, the designated verifier would accept a forged signature created by the adversary instead of the signer. We denote attack of this kind as forgery against rogue key attack (RKA).

A Proposed Fix. The problem comes from the extra power given to the adversary to create malformed public key. The fix suggest in [16] is to require the certification authority to check the validity of the public key before issuing a digital certificate. In terms of modelling, this implies the stronger certified key model in which the users are required to conduct a proof-of-knowledge of his secret key to the CA. As argue in [17], this requires modification of the client and CA functioning software. We propose another way that could withstand this attack in the plain model based on a technique used in multisignautres [17] and batch verification of digital signatures [22]. In a nutshell, $g^{x_{V}}$ is defined to be $\prod_{i \in[n]}\left(g^{x_{V_{i}}}\right)^{h_{i}}$, where $h_{i}=H\left(g^{x_{S}}, g^{x_{V_{1}}}, \ldots, g^{x_{V_{n}}}, m, i\right)$ for a hash function $H$ which shall be modelled as a random oracle. Observe that with this modification, the value $x_{V}$ can still be computed if all the values $x_{V_{i}}$ are known. On the other hand, if one of the secret keys, say $x_{V_{i}}$, is unknown, the value $x_{V}$ cannot be computed since the probability of "canceling" $g^{x_{V_{i}}}$ in the computation of $g^{x_{V}}$ is negligible assuming the values $h_{i}$ are randomly distributed and are only known after the value of the public keys are chosen.

\subsection{Formal Security Definition for Unforgeability Under Rogue Key Attack}

To formally assert the security of our proposed solution, we define a security model which intends to capture attack of this kind. ${ }^{\ddagger}$ We believe a verification query with the target verifier may be of use to the adversary since the adversary might try to submit mal-formed signatures to learn information about the target verifier's verification procedure.

Unforgeability Against Rogue Key Attack. The following game between a challenger $\mathcal{C}$ and an adversary $\mathcal{A}$ formally captures the requirement of $U F-R K A$

Setup $\mathcal{C}$ invokes Setup $\left(1^{\lambda}\right)$ and subsequently Gen() to obtain (param, $\left(\mathrm{pk}_{S}, \mathrm{sk}_{S}\right),\left(\mathrm{pk}_{V}, \mathrm{sk}_{V}\right)$. (param, $\mathrm{pk}_{S}, \mathrm{pk}_{V}$ ) is given to $\mathcal{A}$.

Query $\mathcal{A}$ is allowed to make the following queries:

- Verification Query. $\mathcal{A}$ submits a set of public keys $\mathcal{V}$, a signature $\left(\sigma, \mathcal{V} \cup \mathrm{pk}_{V}\right)$, a message $m$ and receives valid/invalid $\leftarrow \operatorname{Verify}\left(\mathrm{pk}_{S}, \sigma, \mathcal{V} \cup \mathrm{pk}_{V}, m, \mathrm{sk}_{V}\right)$.

- Signature Query. $\mathcal{A}$ submits a message $m$, a set of public keys $\mathcal{V}$ and receives $(\sigma, \mathcal{V}) \leftarrow$ $\left(\mathrm{sk}_{S}, \mathcal{V}, m\right)$. Note that $\mathcal{A}$ can submit an arbitrary set of verifiers of his choice (even a set without $\mathrm{pk}_{V}$ ).

Output $\mathcal{A}$ submits $\left(\sigma^{*}, m^{*}\right)$ and a set of public keys $\mathcal{V}^{*}$ and wins if and only if

1. valid $\leftarrow \operatorname{Verify}\left(\mathrm{pk}_{S}, \sigma^{*}, \mathcal{V}^{*} \cup \mathrm{pk}_{V}, m^{*}, \mathrm{sk}_{V}\right)$.

2. $\mathcal{A}$ has not submitted a Signature Query with input $\left(m^{*}, \mathcal{V}^{*} \cup \mathrm{pk}_{V}\right)$.

\footnotetext{
${ }^{\ddagger}$ While rogue key attack on MDVS is discussed in [16], no formal security model has been proposed to capture such an attack. 
Definition 7 (UF-RKA)

A MDVS scheme is unforgeable under rogue key attack if no PPT adversary wins the above game with non-negligible probability.

We believe UF-RKA for MDVS is a stronger notion compared with the notion Unforgeability.

\subsection{A Concrete Construction}

We present a concrete MDVS scheme from a commonly used two-party ring signature following the generic construction together with our proposed fix.

- Setup. Let $\mathbb{G}=\langle g\rangle$ be a cyclic group of prime order $p$. Output param as $(\mathbb{G}, p, g)$.

- Gen. Choose a hash function $H:\{0,1\}^{*} \rightarrow \mathbb{Z}_{p}$ which will be modelled as a random oracle ${ }^{\S}$. Randomly generate $x \in_{R} \mathbb{Z}_{p}$, compute $g^{x}$. Output pk as $\left(g^{x}, H\right)$ and sk as $x$.

- Sign. On input the signer's key pair $\left(\mathrm{pk}_{S}, \mathrm{sk}_{S}\right)$, a set of designated verifier's public keys $\mathcal{V}=\left\{\mathrm{pk}_{V_{1}}, \ldots, \mathrm{pk}_{V_{n}}\right\}$ and a message $m$, parse $\mathrm{pk}_{S}$ as $\left(Y_{S}, H_{S}\right), \mathrm{sk}_{S}$ as $x_{S}, \mathrm{pk}_{V_{i}}$ as $\left(Y_{V_{i}}, H_{V_{i}}\right)$. Compute $Y=\prod_{i \in[n]} Y_{V_{i}}^{h_{i}}$ where $h_{i}=H_{V_{i}}\left(\mathrm{pk}_{S}, \mathrm{pk}_{V_{1}}, \ldots, \mathrm{pk}_{V_{n}}, m\right)$.

1. Randomly generate $r, c_{2}, z_{2} \in \mathbb{Z}_{p}$, compute $T_{1}=g^{r}, T_{2}=Y^{c_{2}} g^{z_{2}}$.

2. Compute $c=H_{S}\left(T_{1}, T_{2}, \mathrm{pk}_{S}, \mathrm{pk}_{V_{1}}, h_{1}, \ldots, \mathrm{pk}_{V_{n}}, h_{n}, Y, m\right)$ and $c_{1}=c-c_{2}$.

3. Compute $z_{1}=r-c_{1} x_{S}$.

Output the signature as $\left(c_{1}, c_{2}, z_{1}, z_{2}, \mathcal{V}\right)$. Note that $\left(c_{1}, c_{2}, z_{1}, z_{2}\right)$ is a ring signature on message $m$ with respective to ring $\left\{Y_{S}, Y\right\}$.

- We remark that the above steps constitute a standard signature proof-of-knowledge of 1-outof-2 discrete logarithms (which can be viewed as a two-party ring signature). This can be presented as follows using the Camenisch and Stadler notation [23].

$$
\operatorname{SPK}\left\{(\alpha): Y_{S}=g^{\alpha} \vee Y=g^{\alpha}\right\}(m)
$$

- Verify. To verify the signature $\left(c_{1}, c_{2}, z_{1}, z_{2},\left\{\mathrm{pk}_{V_{i}}\right\}_{i \in[n]}\right)$ on message $m$, a verifier parses $\mathrm{pk}_{V_{i}}$ as $\left(Y_{V_{i}}, H_{V_{i}}\right)$ and computes $Y=\prod_{i \in[n]} Y_{V_{i}}^{H_{V_{i}}\left(\mathrm{pk}_{S}, \mathrm{pk}_{V_{1}}, \ldots, \mathrm{pk}_{V_{n}}, m\right)}$. Output valid if and only if

$$
c_{1}+c_{2}=H_{S}\left(Y_{S}^{c_{1}} g^{z_{1}}, Y^{c_{2}} g^{z_{2}}, \mathrm{pk}_{S}, \mathrm{pk}_{V_{1}}, h_{1}, \ldots, \mathrm{pk}_{V_{n}}, h_{n}, Y, m\right)
$$

and invalid otherwise.

Regarding the security of our concrete construction, we have the following theorem, whose proof can be found in Appendix A.

\section{Theorem 1}

Our concrete construction is secure under the discrete logarithm assumption in the random oracle model. Specifically, it satisfies

- definition 4 under the discrete logarithm assumption in the random oracle model;

- definition 5 unconditionally;

- definition 7 under the discrete logarithm assumption in the random oracle model.

\section{GENERIC STRONG MDVS}

Strong DVS can be constructed from DVS via encrypting the signature under the designated verifier's public key. However, the intuitive solution of encrypting the signature under each designated verifier's public key in the case of multiple designated verifiers is not satisfactory. As

\footnotetext{
$\S$ We abuse the notation and assume a full domain hash. In the following when we write $c=H(X, Y)$ where $X$ and $Y$ may be elements from different domains, we assume a suitable encoding scheme is employed to convert $X, Y$ into a bit-string.
} 
discussed in [5], this intuitive solution creates a subtle issue in correctness. Specifically, if some of the encryptions are not executed properly, the signer could create an "invalid" signature that would be regarded as valid by some verifiers.

\subsection{Overview of Our Generic Construction}

To tackle this challenge, we observe that it is straightforward to use a verifiable encryption [24] which allows the signer to create a proof that all ciphertext decrypts to the same value. By verifying the proof, all verifiers are assured that all the verifiers obtains the same value for signature verification. This solution is, however, expensive. Looking at an abstract level, the goal of this encryption is to ensure all verifiers obtains the same value via decryption. This can be achieved, perhaps somewhat interestingly, using a very weak one-way encryption with an explicit "IND-CPA" attack. Denote such an encryption scheme as $\mathcal{W E}$. That is, given a message $k$, anyone can check if the ciphertext $C$ decrypts to it. It is easy for a verifier to check locally if all the encryptions of the designated verifier signature are properly done.

This creates another problem. Since $\mathcal{W E}$ is only one-way secure, the ciphertext might leak information about the signature being encrypted and thus privacy of signer's identity is not guaranteed. Thus, we employ a hybrid approach. $\mathcal{W E}$ is used to encrypt a symmetric key $k$ under all the designated verifiers public keys into ciphertexts $C_{1}, \ldots, C_{n}$. The ordinary MDVS is encrypted with a symmetric key encryption $\mathcal{S E}$ with key $k$. As long as the key $k$ cannot be recovered from the ciphertext $C_{i}$ 's, no information about the MDVS can be learnt as long as the symmetric encryption $\mathcal{S E}$ is secure. Looking ahead, we assume $\mathcal{S E}$ to be an idealized cipher for the ease of security analysis. This means that our generic construction is secure in the ideal cipher model, which is equivalent to the random oracle model due to the result of [25].

\subsection{Building Block of Our Generic Construction}

While conceptually simple, two properties regarding $\mathcal{W E}$ are needed. The first one is an efficient and explicit "IND-CPA" attack. The second one is an efficient and explicit malleability attack which allows anyone to transform a ciphertext $C$ under public key $Y$ into another ciphertext $C^{\prime}$ under public key $Y^{\prime}$ so that they are encrypting the same message. The malleability attack on $\mathcal{W E}$ is needed in the proof of security for multiple designated verifiers.

Below we define the requirement of the weakly secure encryption $\mathcal{W E}$ as follows.

- $\operatorname{param}_{\mathcal{W E}} \leftarrow \mathcal{W E}$.Setup $\left(1^{\lambda}\right)$ : On input a security parameter $\lambda$, this algorithm outputs the public parameter param $\mathrm{WEE}_{\mathcal{E}}$ for the system. We assume param $\mathrm{WEE}_{\mathcal{E}}$ is an implicit input to all algorithms listed below.

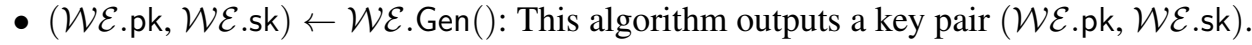

- $C_{\mathcal{W E}} \leftarrow \mathcal{W E}$. $\operatorname{Enc}(\mathcal{W E}$.pk, $m)$ : On input a message $m$ and a public key of the receiver $\mathcal{W} \mathcal{E}$.pk, this algorithm outputs the ciphertext $C_{\mathcal{W E}}$.

- $m \leftarrow \mathcal{W E}$. Dec $\left(\mathcal{W E}\right.$.sk, $\left.C_{\mathcal{W E}}\right):$ On input a secret key $\mathcal{W E}$.sk, a ciphertext $C_{\mathcal{W E}}$, this algorithm outputs the plaintext $m$.

- $0 / 1 \leftarrow \mathcal{W E}$. $\operatorname{iAtk}\left(\mathcal{W E}\right.$.pk, $\left.C_{\mathcal{W E}}, m\right)$ : This is an attack on indistinguishability of ciphertext. On input a public key $\mathcal{W E}$.pk, a ciphertext $C_{\mathcal{W E}}$ and a plaintext $m$, output 1 if and only if $m=\mathcal{W E}$. $\operatorname{Dec}\left(\mathcal{W E}\right.$.sk, $\left.C_{\mathcal{W E}}\right)$, where $\mathcal{W E}$.sk is the corresponding private key of $\mathcal{W E}$.pk and 0 otherwise. Note that $\mathcal{W E}$.sk is not an input to this algorithm.

- $\left(C_{\mathcal{W E}}^{\prime}, \mathcal{W E} . \mathrm{pk}^{\prime}\right) \leftarrow \mathcal{W E}$.mAtk $\left(\mathcal{W E}\right.$.pk, $\left.C_{\mathcal{W E}}\right)$ : This is an attack on malleability of ciphertext. On input a public key $\mathcal{W E}$.pk, a ciphertext $C_{\mathcal{W E}}$, output $C_{\mathcal{W E}}^{\prime}, \mathcal{W E}$.pk $\mathrm{pk}^{\prime}$ such that the distribution of $C_{\mathcal{W E}}^{\prime}$ is indistinguishable to that of $\mathcal{W E}$. $\operatorname{Enc}\left(\mathcal{W E}\right.$.pk ${ }^{\prime}, \mathcal{W E}$. $\operatorname{Dec}\left(\mathcal{W E}\right.$. sk, $\left.\left.C_{\mathcal{W E}}\right)\right)$. Note that the algorithm does not output the corresponding secret key for $\mathcal{W} \mathcal{E}$.pk'.

We require the one-way security of $\mathcal{W E}$, which is formally defined as the following game between a challenger $\mathcal{C}$ and an adversary $\mathcal{A}$.

Setup $\mathcal{C}$ invokes $\mathcal{W E}$.Setup $\left(1^{\lambda}\right)$ and subsequently $\mathcal{W E}$.Gen () to obtain $\left(\operatorname{param}_{\mathcal{W E}}, \mathcal{W E}\right.$.pk, $\mathcal{W} \mathcal{E}$.sk). 
Challenge $\mathcal{C}$ picks a random message $m$, compute $C_{\mathcal{W E}} \leftarrow \mathcal{W E}$.Enc $(\mathcal{W E}$.pk, $m)$. (param WE $_{\mathcal{E}}$, $\mathcal{W E}$.pk, $\left.C_{\mathcal{W E}}\right)$ is given to $\mathcal{A}$.

Output $\mathcal{A}$ outputs $m^{\prime}$ and wins if and only if $m=m^{\prime}$.

$\mathcal{W E}$ is one-way secure if no PPT adversary $\mathcal{A}$ wins the above game with non-negligible probability.

We propose a construction of $\mathcal{W E}$ based on the Elgamal encryption in a cyclic group equipped with a bilinear map.

- $\mathcal{W E}$.Setup $\left(1^{\lambda}\right)$ : Generate a pair of groups $\mathbb{G}, \mathbb{G}_{T}$ of the same prime order $p$ of $\lambda$-bit and a bilinear map $\hat{e}: \mathbb{G} \times \mathbb{G} \rightarrow \mathbb{G}_{T}$. Let $g$ be a generator of $\mathbb{G}$. Set param $\mathcal{W E}=\left(\mathbb{G}, \mathbb{G}_{T}, p, g, \hat{e}\right)$.

- $\mathcal{W E}$.Gen(): Randomly pick $u \in_{R} \mathbb{Z}_{p}$, compute $U=g^{u}$. Set $(\mathcal{W E}$.pk, $\mathcal{W} \mathcal{E}$.sk $)=(U, u)$.

- $\mathcal{W E}$. Enc $(U, m):$ On input a message $m \in \mathbb{G}$, randomly generate $r \in_{R} \mathbb{Z}_{p}$, output $C_{\mathcal{W E}}=$ $(C, D)$ as $\left(m U^{r}, g^{r}\right)$.

- $\mathcal{W E} \cdot \operatorname{Dec}(u,(C, D))$ : Output $C / D^{u}$.

- $\mathcal{W E}$.iAtk $(U,(C, D), m)$ : Output 1 if and only if

$$
\hat{e}(C / m, g)=\hat{e}(D, U)
$$

and 0 otherwise.

- $\mathcal{W E} \cdot \operatorname{mAtk}(U,(C, D))$ : Randomly pick $e, f \in_{R} \mathbb{Z}_{p}$, compute $U^{\prime}=U g^{e}$. Compute $\tilde{C}=C D^{e}$, $C^{\prime}=\tilde{C} U^{\prime f}, D^{\prime}=D g^{f}$. Output $\left(\left(C^{\prime}, D^{\prime}\right), U^{\prime}\right)$.

Note that if $U=g^{u}, C=m U^{r}, D=g^{r}$, it is easy to see that $C^{\prime}=m\left(U g^{e}\right)^{r+f}, D^{\prime}=g^{r+f}$ and $U^{\prime}=U g^{e}$. Thus, $\left(C^{\prime}, D^{\prime}\right)$ is encrypting the message $m$ under the public key $U^{\prime}$ with the correct distribution.

Next, we show that our construction of $\mathcal{W E}$ is one-way secure under the computational DiffieHellman assumption.

Proof

Suppose there exists an adversary $\mathcal{A}$ that can win the game one-way security, we show how to construction an algorithm $\mathcal{S}$ that solves the $\mathrm{CDH}$ problem in a group equipped with a bilinear map. $\mathcal{S}$ is given $\left(\mathbb{G}, \mathbb{G}_{T}, \hat{e}, p, g, g^{a}, g^{b}\right)$ and its goal is to output $g^{a b}$.

$\mathcal{S}$ randomly picks a value $C$, gives param $\mathcal{W E}_{\mathcal{E}}=\left(\mathbb{G}, \mathbb{G}_{T}, p, g, \hat{e}\right), U=g^{a},(C, D)=\left(C, g^{b}\right)$ to $\mathcal{A}$. Note that this implicit set the message being encrypted as $m=C / g^{a b}$. $\mathcal{A}$ returns with a value $m^{\prime} . \mathcal{S}$ computes $C / m^{\prime}$ and outputs it as the solution to the $\mathrm{CDH}$ problem.

\subsection{Our Generic Construction of Strong MDVS}

We present our generic construction of Strong MDVS. Let $\mathcal{M S}=(\mathcal{M S}$.Setup, $\mathcal{M S}$.Gen, $\mathcal{M S}$.Sign, $\mathcal{M S}$.Verify) be a secure MDVS scheme. Let $\mathcal{W E}=(\mathcal{W E}$.Setup, $\mathcal{W E}$.Gen, $\mathcal{W E}$. Enc, $\mathcal{W E}$.Dec, $\mathcal{W E}$.iAtk, $\mathcal{W E}$.mAtk) be a one-way secure encryption. Let $H$ be a hash function and $\mathcal{S E}$ be a symmetric key encryption. We use $\mathcal{S E}$. Enc $c_{k}$ and $\mathcal{S E}$. Dec $_{k}$ to denote encryption and decryption operation of $\mathcal{S E}$ using key $k . H, \mathcal{S E}$ will be modelled as a random oracle and an ideal cipher respectively. We show how to construct a strong MDVS scheme (Setup, Gen, Sign, Verify) as follows.

- Setup. On input security parameter $1^{\lambda}$, invoke param $\mathcal{M S}_{\mathcal{S}} \leftarrow \mathcal{M S}$.Setup $\left(1^{\lambda}\right)$ and param $\mathrm{WE}_{\mathcal{E}} \leftarrow$ $\mathcal{W E}$.Setup $\left(1^{\lambda}\right)$, specify a weak encryption $\mathcal{W E}$, a hash function $H$ and a symmetric cipher $\mathcal{S E}$. Set param $=\left(\right.$ param $_{\mathcal{M S}}$, param $\left._{\mathcal{W E}}, H, \mathcal{S E}\right)$.

- Gen. Invoke $(\mathcal{M S}$.pk, $\mathcal{M S}$.sk $) \leftarrow \mathcal{M S}$.Gen( $),(\mathcal{W E}$.pk, $\mathcal{W} \mathcal{E}$.sk $) \leftarrow \mathcal{W E}$.Gen( $)$. Output pk $=$ $(\mathcal{M S}$.pk, $\mathcal{W E}$.pk $)$ and sk $=(\mathcal{M S}$.sk, $\mathcal{W} \mathcal{E} . \mathrm{sk})$.

- Sign. Let $\mathrm{pk}_{S}=\left(\mathcal{M S} \cdot \mathrm{pk}_{S}, \mathcal{W E} \cdot \mathrm{pk}_{S}\right)$ and $\mathrm{sk}_{S}=\left(\mathcal{M S} \cdot \mathrm{sk}_{S}, \mathcal{W E} \cdot \mathrm{sk}_{S}\right)$ be the key pair of the signer. Let $m$ be the message to be signed. Parse the set of verifiers to 
be $\mathcal{V}=\left\{\mathrm{pk}_{V_{1}}, \ldots, \mathrm{pk}_{V_{n}}\right\}$ such that $\mathrm{pk}_{V_{i}}=\left(\mathcal{M S} \cdot \mathrm{pk}_{i}, \mathcal{W E} \cdot \mathrm{pk}_{i}\right)$. Denote by $\mathcal{V}_{\mathcal{M S}}$ the set $\left\{\mathcal{M S} . \mathrm{pk}_{1}, \ldots, \mathcal{M S} . \mathrm{pk}_{n}\right\}$.

The signer randomly picks $k \in_{R}\{0,1\}^{\lambda}$. For $i=1$ to $n$, compute

$$
C_{i}=\mathcal{W E} \cdot \operatorname{Enc}\left(\mathcal{W E} \cdot \mathrm{pk}_{i}, k\right)
$$

Next, compute $\tau=H\left(\mathrm{pk}_{S}, \mathrm{pk}_{V_{1}}, C_{1}, \ldots, \mathrm{pk}_{V_{n}}, C_{n}, m\right)$. Invoke $\left(\sigma_{\mathcal{M S}}, \quad \mathcal{V}_{\mathcal{M S}}\right) \leftarrow$ $\operatorname{Sign}\left(\mathcal{M S} . \mathrm{sk}_{S}, \mathcal{V}_{\mathcal{M S}}, m \| \tau\right)$. Invoke $E=\mathcal{S E}$. $\operatorname{Enc}_{k}\left(\sigma_{\mathcal{M S}}\|\tau\| \mathrm{pk}_{S}\right)$.

Output the signature as $\left(E, \mathcal{V},\left\{C_{i}\right\}_{i \in[n]}\right)$.

- Verify. To verify a signature $\left(E, \mathcal{V},\left\{C_{i}\right\}_{i \in[n]}\right)$ on message $m$, a verifier $V$ parses $\mathrm{pk}_{V_{i}}$ as $\left(\mathcal{M S}\right.$. $\mathrm{pk}_{i}, \mathcal{W E}$. $\left.\mathrm{pk}_{i}\right)$ for all $\mathrm{pk}_{V_{i}} \in \mathcal{V}$ and uses his secret key $\left(\mathcal{M S}\right.$.sk $\left.{ }_{V}, \mathcal{W E} . \mathrm{sk}_{V}\right)$ as follows.

- Locate the index $i$ such that $\mathrm{pk}_{V}=\mathrm{pk}_{V_{i}}$. Use his secret key to compute $k=\mathcal{V E}$.Dec $\left(C_{i}\right.$, $\left.\mathcal{W E} . \mathrm{sk}_{V}\right)$.

- For all $j \in[n] \backslash\{i\}$, check if $1=\mathcal{W E}$.iAtk $\left(\mathcal{W E} \cdot\right.$ pk $\left._{i}, C_{i}, k\right)$. Output invalid if any of the check outputs 0 .

- Compute $\sigma_{\mathcal{M S}}, \tau, \mathrm{pk}_{S}$ by $\mathcal{S E}$. $\operatorname{Dec}_{k}(E)$.

- Output invalid if $\tau \neq H\left(\mathrm{pk}_{S}, \mathrm{pk}_{V_{1}}, C_{1}, \ldots, \mathrm{pk}_{V_{n}}, C_{n}, m\right)$.

- Parse $\mathrm{pk}_{S}$ as $\left(\mathcal{M S} \cdot \mathrm{pk}_{S}, \mathcal{W E} \cdot \mathrm{pk}_{S}\right)$.

- Invoke valid/ invalid $\leftarrow \mathcal{M S}$.Verify $\left(\mathcal{M S} . \mathrm{pk}_{S}, \sigma_{\mathcal{M S}},\left\{\mathcal{M S} . \mathrm{pk}_{1}, \ldots, \mathcal{M S} . \mathrm{pk}_{n}\right\}\right.$, $\left.m \| \tau, \mathcal{M S} . \mathrm{sk}_{V}\right)$.

Regarding the security of our generic construction, we have the following theorem, whose proof can be found in Appendix A.

\section{Theorem 2}

Our generic construction satisfies definition $d$ if the underlying MDVS scheme $\mathcal{M S}$ satisfies definitions $d$ for $d \in\{4,5,7\}$. Furthermore, our generic construction satisfies definition 6 if $\mathcal{W E}$ is one-way secure in the random oracle model.

\section{OPTIMIZATION OF OUR GENERIC STRONG MDVS}

Following the above idea, the goal of the weak one-way encryption $\mathcal{W E}$ is to encrypt a common symmetric key for all designated verifiers. It can be replaced with a secure broadcast encryption. The correctness of the broadcast encryption would ensure all designated verifiers obtain the same common symmetric key. As in the case for $\mathcal{W E}$, we shall show that this broadcast encryption needs to be one-way secure with an explicit "IND-CPA attack". While it is unlikely to have broadcast encryption with constant-size ciphertext when the public key of the verifiers are generated independently, it is still hopeful to have a construction that is more efficient than encrypting individually for all designated verifiers. We formalise the requirement of this broadcast encryption, denoted as $\mathcal{B E}$, and give an efficient construction. We also show how this can be used to construct a possibly more efficient Strong MDVS.

- $\operatorname{param}_{\mathcal{B E}} \leftarrow \mathcal{B E}$.Setup $\left(1^{\lambda}\right)$ : On input a security parameter $\lambda$, this algorithm outputs the public parameter param $\mathrm{m}_{\mathcal{B E}}$ for the system. We assume param $\mathrm{m}_{\mathcal{B E}}$ is an implicit input to all algorithms listed below.

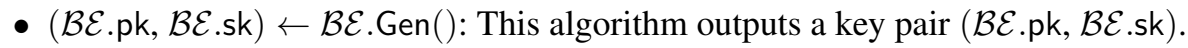

- $\left(C_{\mathcal{B E}}, \mathcal{Y}_{\mathcal{B E}}\right) \leftarrow \mathcal{B E}$. $\operatorname{Enc}\left(\mathcal{Y}_{\mathcal{B E}}, m\right)$ : On input a message $m$ and a set of public keys of the

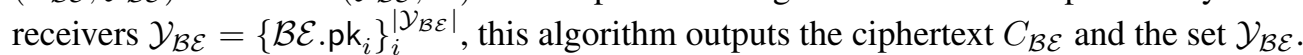

- $m \leftarrow \mathcal{B E}$. Dec $\left(\mathcal{B E}\right.$.sk, $\left.C_{\mathcal{B E}}, \mathcal{Y}_{\mathcal{B E}}\right):$ On input a ciphertext $C_{\mathcal{W E}}$, a receiver set $\mathcal{Y}_{\mathcal{B E}}$, and a secret key $\mathcal{B E}$.sk such that the corresponding public key $\mathcal{B E}$.pk $\in \mathcal{Y}_{\mathcal{B E}}$, this algorithm outputs the plaintext $m$ or $\perp$ for invalid ciphertext.

- $0 / 1 \leftarrow \mathcal{B E}$. $\operatorname{Atk}\left(\mathcal{Y}_{\mathcal{B E}}, C_{\mathcal{W E}}, m\right)$ : This is an attack on indistinguishability of ciphertext. On input a set of public keys $\mathcal{Y}_{\mathcal{B E}}$, a ciphertext $C_{\mathcal{B E}}$ and a plaintext $m$, output 1 if and only if $m=\mathcal{B E}$. Dec $\left(\mathcal{B E}\right.$.sk, $\left.C_{\mathcal{B E}}, \mathcal{Y}_{\mathcal{B E}}\right)$, where $\mathcal{B E}$.sk is a corresponding private key of $\mathcal{B E}$.pk $\in \mathcal{Y}_{\mathcal{B E}}$ and 0 otherwise. Note that $\mathcal{B E}$.sk is not an input to this algorithm. 
One-wayness of $\mathcal{B E}$ which is formally defined as the following game between a challenger $\mathcal{C}$ and an adversary $\mathcal{A}$.

Setup $\mathcal{C}$ invokes $\mathcal{B E}$.Setup $\left(1^{\lambda}\right)$ and subsequently $\mathcal{B E}$.Gen () to obtain param $\mathrm{BE}_{\mathcal{B}}$ and a set of key pairs $\left\{\left(\mathcal{B E} . \mathrm{pk}, \mathcal{B E} . \mathrm{sk}_{1}\right), \ldots,\left(\mathcal{B E} . \mathrm{pk}_{n}, \mathcal{B E} \cdot\right.\right.$ sk $\left.\left._{n}\right)\right\}$. Denote $\mathcal{Y}_{\mathcal{B E}}$ as the set $\left\{\mathcal{B E} . \mathrm{pk}_{i}\right\}_{i=1}^{n}$.

Challenge $\mathcal{C}$ picks a random message $m$, compute $\left(C_{\mathcal{B E}}, \mathcal{Y}_{\mathcal{B E}}\right) \leftarrow \mathcal{B E}$. Enc $\left(\mathcal{Y}_{\mathcal{B E}}, m\right)$. $\left(\right.$ param $\mathrm{m}_{\mathcal{B}}$, $\left.\mathcal{Y}_{\mathcal{B E}}, C_{\mathcal{B E}}\right)$ is given to $\mathcal{A}$.

Output $\mathcal{A}$ outputs $m^{\prime}$ and wins if and only if $m=m^{\prime}$.

$\mathcal{B E}$ is one-way secure if no PPT adversary $\mathcal{A}$ wins the above game with non-negligible probability.

We would like to stress correctness of $\mathcal{B E}$ is a subtle but important issue in our construction of strong MDVS and we formally define the requirement in the following game between a challenger $\mathcal{C}$ and an adversary $\mathcal{A}$.

Challenge $\mathcal{C}$ invokes $\mathcal{B E}$.Setup $\left(1^{\lambda}\right)$ and subsequently $\mathcal{B E}$.Gen () to obtain param $\mathrm{BE}_{\mathcal{B E}}$ and a set of key pairs $\left\{\left(\mathcal{B E}\right.\right.$.pk, $\left.\left.\mathcal{B E} . \mathrm{sk}_{1}\right), \ldots,\left(\mathcal{B E} . \mathrm{pk}_{n}, \mathcal{B E} . \mathrm{sk}_{n}\right)\right\}$. Denote $\mathcal{Y}_{\mathcal{B E}}$ as the set $\left\{\mathcal{B E} . \mathrm{pk}_{i}\right\}_{i=1}^{n}$. $\left(\right.$ param $\left._{\mathcal{B E}}, \mathcal{Y}_{\mathcal{B E}}\right)$ is given to $\mathcal{A}$.

Output $\mathcal{A}$ outputs $C_{\mathcal{B E}} \cdot \mathcal{C}$ computes $m_{i}^{\prime} \leftarrow \mathcal{B E}$. $\operatorname{Dec}\left(\mathcal{B E} . \mathrm{sk}_{i}, C_{\mathcal{B E}}, \mathcal{Y}_{\mathcal{B E}}\right)$ for $i=1$ to $n$. $\mathcal{A}$ wins if and only if there exists $i, j \in[n]$ such that $m_{i}^{\prime} \neq m_{j}^{\prime}$.

$\mathcal{B E}$ is said to be correct if no PPT adversary $\mathcal{A}$ wins the above game with non-negligible probability.

\subsection{A Construction of $\mathcal{B E}$}

We present a simple construction of $\mathcal{B E}$, which is based on the optimization of our $\mathcal{W E}$ with multiple receivers re-using the same randomness.

- $\mathcal{B E}$.Setup $\left(1^{\lambda}\right)$ : Generate a pair of groups $\mathbb{G}_{,} \mathbb{G}_{T}$ of the same prime order $p$ of $\lambda$-bit and a bilinear map $\hat{e}: \mathbb{G} \times \mathbb{G} \rightarrow \mathbb{G}_{T}$. Let $g$ be a generator of $\mathbb{G}$. Set param $\mathcal{B E}=\left(\mathbb{G}, \mathbb{G}_{T}, p, g, \hat{e}\right)$.

- $\mathcal{B E}$.Gen (): Randomly pick $u \in_{R} \mathbb{Z}_{p}$, compute $U=g^{u}$. Set $(\mathcal{B E}$.pk, $\mathcal{B E}$.sk $)=(U, u)$.

- $\mathcal{B E}$. Enc $\left(\left\{U_{1}, \ldots, U_{n}\right\}, m\right)$ : On input a message $m \in \mathbb{G}$, randomly generate $r \in_{R} \mathbb{Z}_{p}$, output $C_{\mathcal{B E}}=\left(C, D_{1}, \ldots, D_{n}\right)$ as $\left(m g^{r}, U_{1}^{r}, \ldots, U_{n}^{r}\right)$.

- $\mathcal{B E} . \operatorname{Dec}\left(u_{i},\left(C, D_{1}, \ldots, D_{n}\right),\left\{U_{1}, \ldots, U_{n}\right\}\right)$ : Compute $m=C / D_{i}^{u_{i}^{-1}}$. Randomly generate $\ell_{1}, \ldots, \ell_{n} \in_{R} \mathbb{Z}_{p}$, output $m$ if and only if

$$
\hat{e}\left(C / m, U_{1}^{\ell_{1}} \cdots U_{n}^{\ell_{n}}\right)=\hat{e}\left(g, D_{1}^{\ell_{1}} \cdots D_{n}^{\ell_{n}}\right)
$$

Output $\perp$ otherwise.

- $\mathcal{B E}$.iAtk $\left(\left\{U_{1}, \ldots, U_{n}\right\},\left(C, D_{1}, \ldots, D_{n}\right), m\right)$ : Randomly generate $\ell_{1}, \ldots, \ell_{n} \in_{R} \mathbb{Z}_{p}$, output 1 if and only if

$$
\hat{e}\left(C / m, U_{1}^{\ell_{1}} \cdots U_{n}^{\ell_{n}}\right)=\hat{e}\left(g, D_{1}^{\ell_{1}} \cdots D_{n}^{\ell_{n}}\right)
$$

and 0 otherwise.

Note that we use the technique in batch verification of pairing-based digital signatures again to assure all verifiers would decrypt to the same value. This can be explained intuitively as follows. If we denote $\hat{r}$ as the discrete logarithm of $C / m$ to base $g$, the equation

$$
\hat{e}\left(C / m, U_{1}^{\ell_{1}} \cdots U_{n}^{\ell_{n}}\right)=\hat{e}\left(g, D_{1}^{\ell_{1}} \cdots D_{n}^{\ell_{n}}\right)
$$

for randomly picked values $\ell_{1}, \ldots \ell_{n}$ implies $\left(U_{1}^{\hat{r}}\right)^{\ell_{1}} \cdots\left(U_{n}^{\hat{r}}\right)^{\ell_{n}}=D_{1}^{\ell_{1}} \cdots D_{n}^{\ell_{n}}$ for all possible values of $\ell_{i}$ with overwhelming probability. This implies $D_{i}=U_{i}^{\hat{r}}$ and thus all receivers decrypt to the same value.

Next, we show that our construction of $\mathcal{B E}$ is one-way secure under the divisible computational Diffie-Hellman assumption (which is equivalent to the $\mathrm{CDH}$ assumption). 


\section{Proof}

Suppose there exists an adversary $\mathcal{A}$ that can win the game one-way security, we show how to construction an algorithm $\mathcal{S}$ that solves the DCDH problem in a group equipped with a bilinear map. $\mathcal{S}$ is given $\left(\mathbb{G}, \mathbb{G}_{T}, \hat{e}, p, g, g^{a}, g^{b}\right)$ and its goal is to output $g^{a / b}$.

$\mathcal{S}$ chooses the size of the set $n$, randomly picks $\alpha_{i} \in_{R} \mathbb{Z}_{p}$ and computes $U_{i}=\left(g^{b}\right)^{\alpha_{i}}$ for $i=1$ to $n$. Denote $\mathcal{Y}_{\mathcal{B E}}$ as the set $\left\{U_{i}\right\}_{i=1}^{n} \cdot \mathcal{S}$ computes $D_{i}=\left(g^{a}\right)^{\alpha_{i}}$ and randomly chooses $C \in_{R} \mathbb{G}$. $\mathcal{S}$ gives param $\operatorname{\mathcal {B}}_{\mathcal{B}}=\left(\mathbb{G}, \mathbb{G}_{T}, p, g, \hat{e}\right), C_{\mathcal{B E}}=\left(C, D_{1}, \ldots, D_{n}\right), \mathcal{Y}_{\mathcal{B E}}$ to $\mathcal{A}$. Denote $r$ as the value $a / b$, it can be seen that the ciphertext given to the adversary is the encryption of the value $m=C / g^{r}$ (it can be seen that $D_{i}=U_{i}^{r}$ for $i=1$ to $n$.).

When the adversary returns $m^{\prime}, \mathcal{S}$ computes $C / m^{\prime}$ as the solution for the DCDH problem. Note that $C / m^{\prime}$ will be $g^{a / b}$.

\subsection{Generic Construction of Strong MDVS from $\mathcal{B E}$}

Here, we use $\mathcal{B E}$ as the building block for the generic construction of strong MDVS. Note that this is possibly better than the generic construction given in Section 4 as the previous generic construction implies that the creation, verification as well as the size of the signature are both linear to the number of designated verifiers. In practice, although our construction using $\mathcal{B E}$ is still linear size, the computation has been reduced from $n$ pairings to $n$ exponentiations where $n$ is the number of designated verifiers. Furthermore, the signature size is reduced by half.

We present our second generic construction of Strong MDVS. Let $\mathcal{M S}=(\mathcal{M S}$.Setup, $\mathcal{M S}$.Gen, $\mathcal{M S}$.Sign, $\mathcal{M S}$.Verify) be a secure MDVS scheme. Let $\mathcal{B E}=(\mathcal{B E}$.Setup, $\mathcal{B E}$.Gen, $\mathcal{B E}$. Enc, $\mathcal{B E}$. Dec, $\mathcal{B E}$.iAtk) be a one-way secure broadcast encryption $\mathcal{B E}$. Let $H$ be a hash function and $\mathcal{S E}$ be a symmetric key encryption. We use $\mathcal{S E}$. Enc $c_{k}$ and $\mathcal{S E}$. Dec ${ }_{k}$ to denote encryption and decryption operation of $\mathcal{S E}$ using key $k . H, \mathcal{S E}$ will be modelled as a random oracle and an ideal cipher respectively. We show how to construct a strong MDVS scheme (Setup, Gen, Sign, Verify) as follows.

- Setup. On input security parameter $1^{\lambda}$, invoke param ${ }_{\mathcal{M S}} \leftarrow \mathcal{M S}$.Setup $\left(1^{\lambda}\right)$ and param $\mathrm{BE}_{\mathcal{B E}} \leftarrow$ $\mathcal{B E}$.Setup $\left(1^{\lambda}\right)$, a hash function $H$ and a symmetric cipher $\mathcal{S E}$. Set param $=\left(\right.$ param $_{\mathcal{M S}}$, $\left.\operatorname{param}_{\mathcal{B E}}, H, \mathcal{S E}\right)$.

- Gen. Invoke $(\mathcal{M S}$.pk, $\mathcal{M S}$.sk $) \leftarrow \mathcal{M S}$.Gen ()$,(\mathcal{B E}$.pk, $\mathcal{B E}$.sk $) \leftarrow \mathcal{B E}$.Gen () . Output pk $=$ $(\mathcal{M S}$.pk, $\mathcal{B E}$.pk $)$ and sk $=(\mathcal{M S}$.sk, $\mathcal{B E}$.sk $)$.

- Sign. Let $\mathrm{pk}_{S}=\left(\mathcal{M S} \cdot \mathrm{pk}_{S}, \mathcal{B E} \cdot \mathrm{pk}_{S}\right)$ and $\mathrm{sk}_{S}=\left(\mathcal{M S} \cdot \mathrm{sk}_{S}, \mathcal{B E} \cdot \mathrm{sk}_{S}\right)$ be the key pair of the signer. Let $m$ be the message to be signed. Parse the set of verifiers to be $\mathcal{V}=\left\{\mathrm{pk}_{V_{1}}, \ldots, \mathrm{pk}_{V_{n}}\right\}$ such that $\mathrm{pk}_{V_{i}}=\left(\mathcal{M S} \cdot \mathrm{pk}_{i}, \mathcal{B E} \cdot \mathrm{pk}_{i}\right)$. Denote by $\mathcal{V}_{\mathcal{M S}}$ the set $\left\{\mathcal{M S} . \mathrm{pk}_{1}, \ldots, \mathcal{M S}\right.$. pk $\left.{ }_{n}\right\}$. Denote by $\mathcal{V}_{\mathcal{B E}}$ the set $\left\{\mathcal{B E} \cdot \mathrm{pk}_{1}, \ldots, \mathcal{B E} . \mathrm{pk}_{n}\right\}$.

The signer randomly picks $k \in_{R}\{0,1\}^{\lambda}$ and computes $\left(C_{\mathcal{B E}}, \mathcal{V}_{\mathcal{B E}}=\mathcal{B E}\right.$. Enc $\left(\mathcal{V}_{\mathcal{B E}}, k\right)$.

Next, compute $\tau=H\left(\mathrm{pk}_{S}, \mathrm{pk}_{V_{1}}, \ldots, \mathrm{pk}_{V_{n}}, C_{\mathcal{B E}}, m\right)$. Invoke $\left(\sigma_{\mathcal{M S}}, \mathcal{V}_{\mathcal{M S}}\right) \leftarrow \operatorname{Sign}\left(\mathcal{M S} . \mathrm{sk}_{S}\right.$, $\left.\mathcal{V}_{\mathcal{M S}}, m \| \tau\right)$. Invoke $E=\mathcal{S E}$. Enc $_{k}\left(\sigma_{\mathcal{M S}}\|\tau\| \mathrm{pk}_{S}\right)$.

Output the signature as $\left(E, \mathcal{V}, C_{\mathcal{B E}}\right)$.

- Verify. To verify a signature $\left(E, \mathcal{V}, C_{\mathcal{B E}}\right)$ on message $m$, a verifier $V$ parses pk $V_{V_{i}}$ as $\left(\mathcal{M S}\right.$.pk ${ }_{i}$, $\left.\mathcal{B E} . \mathrm{pk}_{i}\right)$ for all $\mathrm{pk}_{V_{i}} \in \mathcal{V}$ and uses his secret key $\left(\mathcal{M S} . \mathrm{sk}_{V}, \mathcal{B E} . \mathrm{sk}_{V}\right)$ as follows.

- Locate the index $i$ such that $\mathrm{pk}_{V}=\mathrm{pk}_{V_{i}}$. Use his secret key to compute $k=$ $\mathcal{B E} . \operatorname{Dec}\left(\mathcal{B E}\right.$. sk $\left._{V}, C_{\mathcal{B E}}, \mathcal{V}_{\mathcal{B E}}\right)$, where $\mathcal{V}_{\mathcal{B E}}$ denotes the set $\left\{\mathcal{B E} . \mathrm{pk}_{1}, \ldots, \mathcal{B E} . \mathrm{pk}_{n}\right\}$.

- Compute $\sigma_{\mathcal{M S}}, \tau, \mathrm{pk}_{S}$ by $\mathcal{S E}$. $\operatorname{Dec}_{k}(E)$.

- Output invalid if $\tau \neq H\left(\mathrm{pk}_{S}, \mathrm{pk}_{V_{1}}, \ldots, \mathrm{pk}_{V_{n}}, C_{\mathcal{B E}}, m\right)$.

- Parse $\mathrm{pk}_{S}$ as $\left(\mathcal{M S} \cdot \mathrm{pk}_{S}, \mathcal{B E} \cdot \mathrm{pk}_{S}\right)$.

- Invoke valid/ invalid $\leftarrow \mathcal{M S}$.Verify $\left(\mathcal{M S}\right.$. $\mathrm{pk}_{S}, \sigma_{\mathcal{M S}},\left\{\mathcal{M S} . \mathrm{pk}_{1}, \ldots, \mathcal{M S} . \mathrm{pk}_{n}\right\}$, $\left.m \| \tau, \mathcal{M S} . \mathrm{sk}_{V}\right)$.

Regarding the security of our generic construction, we have the following theorem, whose proof can be found in Appendix A. 


\section{Theorem 3}

Our generic construction satisfies definition $d$ if the underlying MDVS scheme $\mathcal{M S}$ satisfies definitions $d$ for $d \in\{4,5,7\}$. Furthermore, our generic construction satisfies definition 6 if $\mathcal{B E}$ is one-way secure in the random oracle model.

\subsection{Concrete Instantiations}

Our construction of MDVS in Section 3.4 together with our $\mathcal{B E}$ described in this section could be used together to instantiate a concrete strong MDVS secure against rogue key attack. As a corollary of theorem 3, this particular instantiation would be secure under the $\mathrm{CDH}$ assumption in the random oracle model. Signature generation involves $n+3$ single-base exponentiations and no pairing while its verification involves 1 signle-base exponentiations, 2 two-base exponentiations, $2 n$-base exponentiations and 2 pairings. It is commonly accepted multi-base exponentiations of up to 3 bases could be computed in time comparable with a single-base exponentiation. Its fair to say signature verification would take roughly the same time as $n+3$ single-base exponentiations plus 2 pairing operations.

\section{CONCLUSION}

In this paper, we formalized the security notion unforgeability under rogue key attack for MDVS. We proposed an efficient construction that is provably secure in the proposed model. In addition, we present a generic transformation that converts any secure MDVS scheme into a strong MDVS scheme. We leave the construction of constant size strong MDVS scheme secure under our definitions as an open problem.

\section{REFERENCES}

1. Jakobsson M, Sako K, Impagliazzo R. Designated Verifier Proofs and Their Applications. EUROCRYPT, Lecture Notes in Computer Science, vol. 1070, Maurer UM (ed.), Springer, 1996; 143-154.

2. Chaum D. Private Signature and Proof Systems 1996. US Patent 5,493,614.

3. Saeednia S, Kremer S, Markowitch O. An Efficient Strong Designated Verifier Signature Scheme. ICISC, Lecture Notes in Computer Science, vol. 2971, Lim JI, Lee DH (eds.), Springer, 2003; 40-54.

4. Desmedt Y. Verifier-Designated Signatures. CRYPTO Rump Session 2003; .

5. Laguillaumie F, Vergnaud D. Multi-Designated Verifiers Signatures. ICICS, Lecture Notes in Computer Science, vol. 3269, Lopez J, Qing S, Okamoto E (eds.), Springer, 2004; 495-507.

6. Ng CY, Susilo W, Mu Y. Universal Designated Multi Verifier Signature Schemes. ICPADS (2), IEEE Computer Society, 2005; 305-309.

7. Shailaja G, Kumar KP, Saxena A. Universal Designated Multi Verifier Signature without Random Oracles. ICIT, Mohanty SP, Sahoo A (eds.), IEEE Computer Society, 2006; 168-171.

8. Chow SSM. Identity-Based Strong Multi-Designated Verifiers Signatures. EuroPKI, Lecture Notes in Computer Science, vol. 4043, Atzeni AS, Lioy A (eds.), Springer, 2006; 257-259.

9. Laguillaumie F, Vergnaud D. Multi-Designated Verifiers Signatures: Anonymity without Encryption. Inf. Process. Lett. 2007; 102(2-3):127-132.

10. Li Y, Susilo W, Mu Y, Pei D. Designated Verifier Signature: Definition, Framework and New Constructions. UIC, Lecture Notes in Computer Science, vol. 4611, Indulska J, Ma J, Yang LT, Ungerer T, Cao J (eds.), Springer, 2007; 1191-1200.

11. Chow SSM. Multi-Designated Verifiers Signatures Revisited. I. J. Network Security 2008; 7(3):348-357.

12. Vergnaud D. New Extensions of Pairing-based Signatures into Universal (Multi) Designated Verifier Signatures. CoRR 2008; abs/0802.1076.

13. Chang TY. An ID-Based Multi-Signer Universal Designated Multi-Verifier Signature Scheme. Inf. Comput. 2011; 209(7):1007-1015. URL http: / / dblp.uni-trier.de/db/journals/iandc/iandc20 9. html\#Chang11.

14. Tian H. A New Strong Multiple Designated Verifiers Signature for Broadcast Propagation. INCoS, Xhafa F, Barolli L, Köppen M (eds.), IEEE, 2011; 268-274.

15. Tian H. A New Strong Multiple Designated Verifiers Signature. IJGUC 2012; 3(1):1-11.

16. Shim KA. Rogue-key Attacks on the Multi-designated Verifiers Signature Scheme. Inf. Process. Lett. 2008; 107(2):83-86.

17. Bellare M, Neven G. Multi-Signatures in the Plain Public-Key Model and a General Forking Lemma. ACM Conference on Computer and Communications Security, Juels A, Wright RN, di Vimercati SDC (eds.), ACM, 2006; 390-399.

18. Bao F, Deng RH, Zhu H. Variations of diffie-hellman problem. ICICS, Lecture Notes in Computer Science, vol. 2836, Qing S, Gollmann D, Zhou J (eds.), Springer, 2003; 301-312. 
19. Huang Q, Yang G, Wong DS, Susilo W. Efficient Strong Designated Verifier Signature Schemes without Random Oracle or with Non-Delegatability. Int. J. Inf. Sec. 2011; 10(6):373-385.

20. Laguillaumie F, Vergnaud D. Designated Verifier Signatures: Anonymity and Efficient Construction from Any Bilinear Map. SCN, Lecture Notes in Computer Science, vol. 3352, Blundo C, Cimato S (eds.), Springer, 2004; $105-119$.

21. Rivest RL, Shamir A, Tauman Y. How to Leak a Secret. ASIACRYPT, Lecture Notes in Computer Science, vol. 2248, Boyd C (ed.), Springer, 2001; 552-565.

22. Bellare M, Garay JA, Rabin T. Fast Batch Verification for Modular Exponentiation and Digital Signatures. EUROCRYPT, Lecture Notes in Computer Science, vol. 1403, Nyberg K (ed.), Springer, 1998; 236-250.

23. Camenisch J, Stadler M. Efficient Group Signature Schemes for Large Groups (Extended Abstract). CRYPTO, Lecture Notes in Computer Science, vol. 1294, Jr BSK (ed.), Springer, 1997; 410-424.

24. Camenisch J, Shoup V. Practical Verifiable Encryption and Decryption of Discrete Logarithms. CRYPTO, Lecture Notes in Computer Science, vol. 2729, Boneh D (ed.), Springer, 2003; 126-144.

25. Coron JS, Patarin J, Seurin Y. The random oracle model and the ideal cipher model are equivalent. CRYPTO, Lecture Notes in Computer Science, vol. 5157, Wagner D (ed.), Springer, 2008; 1-20.

\section{A. SECURITY ANALYSIS}

\section{A.1. Proof of Theorem 1}

We prove theorem 1 by the following three lemmas.

Lemma 1 (Unforgeability)

Our construction of MDVS satisfies definition 4 under the discrete logarithm assumption in the random oracle model.

Proof

We prove by reduction. Suppose there exists a PPT adversary $\mathcal{A}$ that wins in game Unforgeability with probability $\epsilon$, we show how to construct a simulator $\mathcal{S}$ that solves the discrete logarithm problem with probability at least $\frac{(n-1) \epsilon}{n}\left(\frac{(n-1) \epsilon}{n q_{H}}-\frac{1}{p}\right)$, where $q_{H_{S}}$ is the number of hash query made by $\mathcal{A}$ to the random oracle $H_{S}$ and $n$ is the number of designated verifiers.

$\mathcal{S}$ is given a problem instance $(\mathbb{G}, g, p, Z)$ and its goal is to compute $z \in \mathbb{Z}_{p}$ such that $Z=g^{z}$. $\mathcal{S}$ plays the role of the challenger $\mathcal{C}$ to the adversary $\mathcal{A}$.

Setup $\mathcal{S}$ sets param as $(\mathbb{G}, g, p)$, randomly picks an integer $n$, a value $x_{i} \in_{R} \mathbb{Z}_{p}$ and a hash function $H_{i}$ for $i=1$ to $n$. Pick a random index $i^{*} \in[n]$. For $i \in[n] \backslash\left\{i^{*}\right\}$, set pk $V_{V_{i}}$ as $\left(Y_{V_{i}}:=g^{x_{i}}, H_{i}\right)$. Set $\mathrm{pk}_{i^{*}}$ as $\left(Y_{V_{i}^{*}}:=Z^{x_{i}^{*}}, H_{i^{*}}\right)$. Pick a hash function $H_{S}$ and set $\mathrm{pk}_{S}$ as $\left(Y_{S}:=Z, H_{S}\right)$. Denote by $\mathcal{V}$ the set $\left\{\mathrm{pk}_{V_{i}}\right\}_{i \in[n]} . \mathcal{S}$ gives $\left(\right.$ param, $\left.\mathrm{pk}_{S}, \mathcal{V}\right)$ to $\mathcal{A}$.

Query $\mathcal{A}$ is allowed to make the following queries:

- Hash Queries. For all hash queries to $H_{S}, \mathcal{S}$ answers with a random value while maintaining consistency. Note that we only need to assume $H_{S}$ is a random oracle in this proof.

- Corruption Query. On input a public key $\mathrm{pk}_{V} \in \mathcal{V}$, locate the index $i$ such that $\mathrm{pk}_{V}=$ $\mathrm{pk}_{V_{i}}$. If $i=i^{*}, \mathcal{S}$ aborts. Otherwise, $\mathcal{S}$ returns $x_{i}$.

- Signature Query. $\mathcal{A}$ submits a message $m . \mathcal{S}$ simulates the signature as follows.

1. $\mathcal{S}$ computes $h_{i}=H_{V_{i}}\left(\mathrm{pk}_{S}, \mathrm{pk}_{V_{1}}, \ldots, \mathrm{pk}_{V_{n}}, m\right)$ and $Y=\prod_{i \in[n]} Y_{V_{i}}^{h_{i}}$.

2. $\mathcal{S}$ randomly picks $c_{1}, c_{2}, z_{1}, z_{2} \in \in_{R} \mathbb{Z}_{p}$, assigns the value $c_{1}+c_{2}$ to the hash query of $H_{S}\left(Y_{S}^{c_{1}} g^{z_{1}}, Y^{c_{2}} g^{z_{2}}, \mathrm{pk}_{S}, \mathrm{pk}_{V_{1}}, h_{1}, \ldots, \mathrm{pk}_{V_{n}}, h_{n}, Y, m\right)$. If the tuple $\left(Y_{S}^{c_{1}} g^{z_{1}}\right.$, $\left.Y^{c_{2}} g^{z_{2}}, \mathrm{pk}_{S}, \mathrm{pk}_{V_{1}}, h_{1}, \ldots, \mathrm{pk}_{V_{n}}, h_{n}, Y, m\right)$ already exists in the set of input query for $H_{S}, \mathcal{S}$ picks another set of $\left(c_{1}, c_{2}, z_{1}, z_{2}\right)$.

3. $\mathcal{S}$ returns $\left(c_{1}, c_{2}, z_{1}, z_{2}, \mathcal{V}\right)$ to $\mathcal{A}$.

Output The probability of not aborting is $\frac{n-1}{n}$ and thus the probability that $\mathcal{A}$ submits a valid signature is $\frac{(n-1) \epsilon}{n}$. Due to the general forking lemma[17], $\mathcal{S}$ can invoke a forking algorithm associated with $\mathcal{A}$ and obtains two valid signatures $\left(c_{1}, c_{2}, z_{1}, z_{2}, \mathcal{V}, m\right)$ and $\left(c_{1}^{\prime}, c_{2}^{\prime}, z_{1}^{\prime}, z_{2}^{\prime}, \mathcal{V}, m\right)$ such that $c_{1}+c_{2} \neq c_{1}^{\prime}+c_{2}^{\prime}$ with probability at least $\frac{(n-1) \epsilon}{n}\left(\frac{(n-1) \epsilon}{n q_{H}}-\frac{1}{p}\right)$. 
Solving the Hard Problem Since $c_{1}+c_{2} \neq c_{1}^{\prime}+c_{2}^{\prime}$, either $c_{1} \neq c_{1}^{\prime}$ or $c_{2} \neq c_{2}^{\prime}$ is true. $\mathcal{S}$ solves the discrete logarithm problem in each case as follows.

- $c_{1} \neq c_{1}^{\prime}$ : By standard argument, $Y_{S}^{c_{1}} g^{z_{1}}=Y_{S}^{c_{1}^{\prime}} g^{z_{1}^{\prime}}$. This implies $Z=g^{\frac{z_{1}^{\prime}-z_{1}}{c_{1}-c_{1}^{\prime}}} \cdot \mathcal{S}$ returns $z=\frac{z_{1}^{\prime}-z_{1}}{c_{1}-c_{1}^{\prime}}$ as the solution to the discrete logarithm problem.

- $c_{2} \neq c_{2}^{\prime}$ : Likewise, $Y^{c_{2}} g^{z_{2}}=Y^{c_{2}^{\prime}} g^{z_{2}^{\prime}}$. S first compute $X=\frac{z_{1}^{\prime}-z_{1}}{c_{1}-c_{1}^{\prime}}$ such that $Y=g^{X}$. Since $Y=\prod_{i \in[n]} Y_{V_{i}}^{h_{i}}, X=\left(\sum_{i \in[n] \backslash\left\{i^{*}\right\}} h_{i} x_{i}\right)+z h_{i^{*}} x_{i^{*}}$, where $z$ is the discrete logarithm of $Z$ to the base $g . \mathcal{S}$ returns $\frac{X-\left(\sum_{i \in[n] \backslash\left\{i^{*}\right\}} h_{i} x_{i}\right)}{\left(h_{i}^{*} x_{i^{*}}\right)}$ as the solution to the discrete logarithm problem.

Lemma 2 (Source Hiding)

Our construction of MDVS satisfies definition 5 unconditionally.

Proof

We describe an algorithm Sim. We follow the view in [11] in which this algorithm should be distributed such that verifiers do not disclose their secret keys to other verifiers.

Sim. On input the signer's public key $\mathrm{pk}_{S}=\left(Y_{S}, H_{S}\right)$, a set of designated verifier's public key $\mathcal{V}=\left\{\mathrm{pk}_{V_{1}}, \ldots, \mathrm{pk}_{V_{n}}\right\}$ and a message $m$, each individual verifier conduct the following:

1. For $i=1$ to $n$, parse the public key $\mathrm{pk}_{V_{i}}$ as $\left(Y_{V_{i}}, H_{V_{i}}\right)$, compute $h_{i}=H_{V_{i}}\left(\mathrm{pk}_{S}, \mathrm{pk}_{V_{1}}, \ldots\right.$, $\left.\mathrm{pk}_{V_{n}}, m\right)$ and $Y=\prod_{i \in[n]} Y_{V_{i}}^{h_{i}}$.

2. Verifier $V_{i}$ randomly generates $r_{i}, c_{1, i}, z_{1, i} \in \mathbb{Z}_{p}$, computes $T_{2, i}=g^{r_{i}}, T_{1, i}=Y_{S}{ }^{c_{1, i}} g^{z_{1, i}}$ and broadcasts $T_{1, i}, T_{2, i}, c_{1, i}, z_{1, i}$ to all other verifiers.

3. Upon receiving all the $T_{1, i}, T_{2, i}$ for $i \in[n]$, all verifiers compute locally $T_{1}=\prod_{i \in[n]} T_{1, i}$, $T_{2}=\prod_{i \in[n]} T_{2, i}, c_{1}=\sum_{i \in[n]} c_{1, i}$ and $z_{1}=\sum_{i \in[n]} z_{1, i}$.

4. All verifiers compute locally $c=H_{S}\left(T_{1}, T_{2}, \mathrm{pk}_{S}, \mathrm{pk}_{V_{1}}, h_{1}, \ldots, \mathrm{pk}_{V_{n}}, h_{n}, Y, m\right)$ and $c_{2}=c-$ $c_{1}$.

5. Verifier $V_{i}$ computes $z_{2, i}=r_{i}-c_{2} h_{i} x_{V_{i}}$, where $x_{V_{i}}$ is the secret key of the verifier $V_{i}$, and broadcasts the value $z_{2, i}$.

6. Upon receiving all the $z_{2, i}$ for $i \in[n]$ from other verifiers, all verifiers compute locally $z_{2}=\sum_{i \in[n]} z_{2, i}$

7. All verifiers can output locally the signature as $\left(c_{1}, c_{2}, z_{1}, z_{2}, \mathcal{V}\right)$.

The distribution of the signature outputted by Sim is identical to that outputted by Sign and thus source hiding holds unconditionally.

Lemma 3 (Unforgeability Against Rogue Key Attack)

Our construction of MDVS satisfies definition 7 under the DL assumption in the random oracle model.

\section{Proof}

We prove by reduction. Suppose there exists a PPT adversary $\mathcal{A}$ that wins in game Unforgeability Against Rogue Key Attack with probability $\epsilon$, we show how to construct a simulator $\mathcal{S}$ that solves the discrete logarithm problem with probability at least $\frac{\epsilon^{4}}{q_{H_{S}}^{2} q_{H_{V}}}-\frac{1}{p}\left(\frac{2+q_{H_{V}}}{q_{H_{S}} q_{H_{V}}}\right)$, where $q_{H_{S}}, q_{H_{V}}$ are the number of hash queries made by $\mathcal{A}$ to the random oracles $H_{S}$ and $H_{V}$ respectively.

$\mathcal{S}$ is given a problem instance $(\mathbb{G}, g, p, Z)$ and its goal is to compute $z \in \mathbb{Z}_{p}$ such that $Z=g^{z}$. $\mathcal{S}$ plays the role of the challenger $\mathcal{C}$ to the adversary $\mathcal{A}$.

Setup $\mathcal{S}$ sets param as $(\mathbb{G}, g, p)$, randomly picks $w \in_{R} \mathbb{Z}_{p}$ and two hash functions $H_{S}, H_{V}$. Parse $\mathrm{pk}_{S}$ and $\mathrm{pk}_{V}$ as $\left(Y_{S}:=Z, H_{S}\right)$ and $\left(Y_{V}:=Z^{w}, H_{V}\right)$ respectively. $\mathcal{S}$ gives (param, pk $\left.{ }_{S}, \mathcal{V}\right)$ to $\mathcal{A}$. 
Query $\mathcal{A}$ is allowed to make the following queries:

- Hash Queries. For each hash query to $H_{S}$ or $H_{V}, \mathcal{S}$ answers with a new random value while maintaining consistency.

- Verification Query. Verification in our construction does not require the secret key and can thus be simulated perfectly.

- Signature Query. $\mathcal{A}$ submits a message $m$ and a set of public keys $\mathcal{V}$. $\mathcal{S}$ simulates the signature as follows.

1. Let $\ell=|\mathcal{V}|$. $\mathcal{S}$ parses $\mathcal{V}$ as $\left\{\mathrm{pk}_{1}:=\left(Y_{1}, H_{1}\right), \ldots, \mathrm{pk}_{\ell}:=\left(Y_{\ell}, H_{\ell}\right)\right\}$.

2. $\mathcal{S}$ computes $h_{j}=H_{j}\left(\mathrm{pk}_{S}, \mathrm{pk}_{1}, \ldots, \mathrm{pk}_{\ell}, m\right)$ and $Y=\prod_{j \in[\ell]} Y_{j}^{h_{j}}$.

3. $\mathcal{S}$ randomly picks $c_{1}, c_{2}, z_{1}, z_{2} \in R \mathbb{Z}_{p}$, assigns the value $c_{1}+c_{2}$ to the hash query of $H_{S}\left(Y_{S}^{c_{1}} g^{z_{1}}, Y^{c_{2}} g^{z_{2}}, \mathrm{pk}_{S}, \mathrm{pk}_{1}, h_{1}, \ldots, \mathrm{pk}_{\ell}, h_{\ell}, Y, m\right)$. If the tuple $\left(Y_{S}^{c_{1}} g^{z_{1}}, Y^{c_{2}} g^{z_{2}}\right.$, $\left.\mathrm{pk}_{S}, \mathrm{pk}_{1}, h_{1}, \ldots, \mathrm{pk}_{\ell}, h_{\ell}, Y, m\right)$ already exists in the input query for $H_{S}, \mathcal{S}$ picks another set of $\left(c_{1}, c_{2}, z_{1}, z_{2}\right)$.

4. $\mathcal{S}$ returns $\left(c_{1}, c_{2}, z_{1}, z_{2}, \mathcal{V}\right)$ to $\mathcal{A}$.

Output $\mathcal{A}$ submits a valid forgery $\left(\sigma^{*}, m^{*}\right)$ and a set of verifiers $\mathcal{V}^{*}=\left\{\mathrm{pk}_{V_{1}}, \ldots, \mathrm{pk}_{V_{n^{*}}}\right\}$. Treating $H_{S}$ as a random oracle, $\mathcal{S}$ can invoke a forking algorithm associated with $\mathcal{A}$ with respect to random oracle $H_{S}$ and obtains two valid signatures $\sigma_{1}:=\left(c_{1}, c_{2}, z_{1}, z_{2}\right.$, $\left.\mathcal{V}^{*}, m^{*}\right)$ and $\sigma_{2}:=\left(c_{1}^{\prime}, c_{2}^{\prime}, z_{1}^{\prime}, z_{2}^{\prime}, \mathcal{V}^{*}, m^{*}\right)$ such that $c_{1}+c_{2} \neq c_{1}^{\prime}+c_{2}^{\prime}$. Denote the value $Y=\prod_{i \in\left[n^{*}\right]} Y_{V_{i}}^{H_{V_{i}}\left(\mathrm{pk}_{S}, \mathrm{pk}_{V_{1}}, \ldots, \mathrm{pk}_{V_{n}}, m\right)}$. Using the same argument as in the proof of Lemma 1, $\mathcal{S}$ obtains a value $z$ such that $Y_{S}=g^{z}$ or $Y=g^{z}$. In the former case, $\mathcal{S}$ successfully solves the discrete logarithm problem. Next, show how $\mathcal{S}$ solves the discrete logarithm problem in the latter situation.

The Second Rewind To solve the discrete logarithm in the second case, $\mathcal{S}$ makes another rewind simulation to the point when $\mathcal{A}$ issues a $H_{V}$ query for $H_{V}\left(\mathrm{pk}_{S}, \mathrm{pk}_{1}, \ldots, \mathrm{pk}_{\ell}, m^{*}\right)$ associated with the forged signature. $\mathcal{S}$ invokes the forking algorithm associated with $\mathcal{A}$ with respect to random oracle $H_{V}$ to obtain another forged signature $\sigma_{3}:=\left(\tilde{c}_{1}, \tilde{c}_{2}, \tilde{z}_{1}, \tilde{z}_{2}, \mathcal{V}^{*}, m^{*}\right)$. After that, $\mathcal{S}$ makes another rewind simulation with respect to random oracle $H_{S}$ again to obtain $\sigma_{4}:=$ $\left(\hat{c}_{1}, \hat{c}_{2}, \hat{z}_{1}, \hat{z}_{2}, \mathcal{V}^{*}, m^{*}\right)$. Since the output of the $H_{V}$ query is an input to the random oracle $H_{S}$ in the forged signature, $\mathcal{A}$ must have made the $H_{V}$ query before marking the query to $H_{S}$. Thus, $\sigma_{3}$ and $\sigma_{4}$ will involve the same set of public keys compared with $\sigma_{1}$ and $\sigma_{2}$. Further more, the value $H_{V}\left(\mathrm{pk}_{S}, \mathrm{pk}_{1}, \ldots, \mathrm{pk}_{\ell}, m^{*}\right)$ in $\left(\sigma_{1}, \sigma_{2}\right)$ (say, $\left.h_{V}\right)$ is different to the value in the signatures $\left(\sigma_{3}, \sigma_{4}\right)$ (say, $\left.h_{V}^{\prime}\right)$. Thus, the value $Y^{\prime}=\prod_{i \in\left[n^{*}\right]} Y_{V_{i}}^{H_{V_{i}}\left(\mathrm{pk}_{S}, \mathrm{pk}_{V_{1}}, \ldots, \mathrm{pk}_{V_{n}}, m\right)}$ is different to $Y$. With signatures $\left(\sigma_{3}, \sigma_{4}\right), \mathcal{S}$ can compute the value $z^{\prime}$ such that $Y_{S}=$ $g^{z^{\prime}}$ or $Y^{\prime}=g^{z^{\prime}}$. If the former is true, $\mathcal{S}$ solves the discrete logarithm problem directly. Otherwise, the value $\left(Y / Y^{\prime}\right)=g^{z-z^{\prime}}$. Recall that $\frac{Y}{Y^{\prime}}=Z^{w h_{V}-w h_{V}^{\prime}}$. Thus, $\mathcal{S}$ can output $\left(z-z^{\prime}\right)\left(w\left(h_{V}-h_{V}^{\prime}\right)\right)^{-1}$ as the solution to the discrete logarithm problem.

Probability Analysis The probability of $\mathcal{A}$ submitting a valid forgery is $\epsilon$. The success probability of invoking the forking algorithm associated with $\mathcal{A}$, denoted as $\mathcal{F}_{\mathcal{A}}$, with respect to the random oracle $H_{S}$ is at least $\epsilon^{\prime}:=\epsilon\left(\frac{\epsilon}{q_{H_{S}}}-\frac{1}{p}\right)$ due to the general forking lemma [17]. The success probability of invoking the forking algorithm associated with $\mathcal{F}_{\mathcal{A}}$ with respect to random oracle $H_{V}$ is at least $\epsilon^{\prime}\left(\frac{\epsilon^{\prime}}{q_{H_{V}}}-\frac{1}{p}\right)$, again due to the general forking lemma [22]. The overall success probability of $\mathcal{S}$ is thus bounded below by

$$
\begin{aligned}
\epsilon^{\prime}\left(\frac{\epsilon^{\prime}}{q_{H_{V}}}-\frac{1}{p}\right) & =\frac{\epsilon^{\prime 2}}{q_{H_{V}}}-\frac{\epsilon^{\prime}}{p} \\
& =\frac{\epsilon^{2}\left(\epsilon / q_{H_{S}}-1 / p\right)^{2}}{q_{H_{V}}}-\frac{\epsilon}{p}\left(\frac{\epsilon}{q_{H_{S}}}-\frac{1}{p}\right) \\
& \geq \frac{\epsilon^{4}}{q_{H_{S}}^{2} q_{H_{V}}}-\frac{2 \epsilon^{3}}{q_{H_{S}} q_{H_{V}} p}-\frac{\epsilon^{2}}{q_{H_{S}} p} \\
& \geq \frac{\epsilon^{4}}{q_{H_{S}}^{2} q_{H_{V}}}-\frac{2+q_{H_{V}}}{p q_{H_{S}} q_{H_{V}}}
\end{aligned}
$$




\section{A.2. Proof of Theorem 2}

We sketch the proof of theorem 2 as follows.

Proof

The proof that our generic construction satisfies definitions 4,5,7 if the underlying MDVS $\mathcal{M S}$ satisfies the corresponding definitions is straightforward. We can easily construct a simulator $\mathcal{S}$ that acts as an attacker against $\mathcal{M S}$ if there exists an adversary $\mathcal{A}$ against the generic construction. $\mathcal{S}$ receives the system parameters of $\mathcal{M S}$, creates the public and secret keys of a one-way encryption $\mathcal{W E}$ for all users and gives them to $\mathcal{A}$. For any verification query submitted by $\mathcal{A}, \mathcal{S}$ can use the secret key of the $\mathcal{W E}$ to translate it to an appropriate query to the $\mathcal{M S}$. Finally, when $\mathcal{A}$ submits a forgery, $\mathcal{S}$ can decrypts it to produce the forgery of the underlying $\mathcal{M S}$.

We focus on the proof against privacy of signer's identity. Let $\mathcal{A}$ be an adversary with nonnegligible advantage in game PSI. We show how to construct a simulator $\mathcal{S}$ that breaks the one-way security of the encryption $\mathcal{W E}$ in the ideal cipher model. The ideal cipher model is equivalent to the random oracle model [25] and is thus subsumed into the random oracle model in the theorem statement. $\mathcal{S}$ is given an instance of $\mathcal{W E}=\left(\operatorname{param}_{\mathcal{W E}}, \mathcal{W E}\right.$.pk $)$ and a challenge ciphertext $C_{\mathcal{W E}}$ and its goal is to output the corresponding plaintext. $\mathcal{S}$ creates the parameter of $\mathcal{M S}$ honestly and is in possession of the keys for the two signers and the set of verifiers, say $\left(\mathcal{M S} . \mathrm{pk}_{i}, \mathcal{M S} . \mathrm{sk}_{i}\right)$ for verifier $V_{i} . \mathcal{S}$ invokes $\mathcal{W E}$.mAtk $\left(\mathcal{W E}\right.$.pk, $\left.C_{\mathcal{W E}}\right)$ repeatedly to obtain a set of public keys and ciphertexts $\left(C_{\mathcal{W E}, i}^{*}, \mathcal{W E} \cdot \mathrm{pk}_{i}\right)$. Set the public keys of the verifiers as $\left(\mathcal{M S} . \mathrm{pk}_{i}, \mathcal{W} \mathcal{E} . \mathrm{pk}_{i}\right)$ for verifier $V_{i} . \mathcal{S}$ chooses a symmetric cipher $\mathcal{S E}$ and a hash function $H . \mathcal{S E}$ is modelled as an idealized cipher.

Setup $\mathcal{S}$ gives $\left(\mathcal{M S}\right.$.param, param $\left.{ }_{\mathcal{W E}}, \mathcal{S E}, H\right)$ to $\mathcal{A}$ as param, together with the public keys of the signers and verifiers.

Query $\mathcal{S}$ uses the secret key of the signers to answer signature queries. Other types of queries are discussed below.

- For verification query of signature $\left(E, \mathcal{V},\left\{C_{i}\right\}_{i \in[n]}\right)$ on message $m, \mathcal{S}$ looks through the encryption query on $\mathcal{S E}$ with key $k$ that produces $E$. If the query is not found, return invalid. Otherwise, $\mathcal{S}$ checks if $1=\mathcal{W E}$. $\operatorname{Atk}\left(\mathcal{W E}\right.$. $\left.\mathrm{pk}_{i}, C_{i}, k\right)$ for all $i=1$ to $n$. If not, $\mathcal{S}$ returns invalid. $\mathcal{S}$ obtains $\left(\sigma_{\mathcal{M S}}, \tau\right.$, pk $\left._{S}\right)$ from the encryption query of $\mathcal{S E}$ with output $E$. $\mathcal{S}$ returns invalid if $\tau \neq H\left(\mathrm{pk}_{S}, \mathrm{pk}_{V_{1}}, C_{1}, \ldots, \mathrm{pk}_{V_{n}}, C_{n}, m\right)$. Otherwise, $\mathcal{S}$ returns the verification results of $\sigma_{\mathcal{M S}}$ with secret key $\mathcal{M S}$.sk ${ }_{i}$.

- For each encryption or decryption query submitted by $\mathcal{A}$ with key $k, \mathcal{S}$ invokes $0 / 1 \leftarrow$ $\mathcal{W E}$.iAtk $\left(\mathcal{W E}\right.$.pk, $\left.C_{\mathcal{W E}}, k\right)$. If the result is $1, \mathcal{S}$ returns $k$ and break the one-way security of $\mathcal{W E}$.

Challenge At some point $\mathcal{A}$ submits a message $m^{*}$. $\mathcal{S}$ flips a fair coin $b \in\{0,1\}$ and computes $\tau=$ $H\left(\mathrm{pk}_{S_{b}}, \mathrm{pk}_{V_{1}}, C_{\mathcal{W E}, 1}^{*}, \ldots, \mathrm{pk}_{V_{n}}, C_{\mathcal{W E}, n}^{*}, m\right)$. Invoke $\left(\sigma_{\mathcal{M S}}, \mathcal{V}_{\mathcal{M S}}\right) \leftarrow \operatorname{Sign}\left(\mathcal{M S} . \mathrm{sk}_{S_{b}}, \mathcal{V}_{\mathcal{M S}}\right.$, $\left.m^{*} \| \tau\right)$. Pick a random value $E^{*}$. Return $\left(E^{*}, \mathcal{V},\left\{C_{\mathcal{W E}, i}^{*}\right\}_{i \in[n]}\right)$ as the challenge signature. This implicitly sets $E^{*}=\mathcal{S E}$. Enc $_{k}\left(\sigma_{\mathcal{M S}}\|\tau\| \mathrm{pk}_{S_{b}}\right)$ where $k$ is the value encrypted in $C_{\mathcal{W E}, i}^{*}$ for $i=1$ to $n$.

Query Same as the previous query phase.

Output $\mathcal{A}$ outputs a guess bit $b^{\prime}$.

It remains to argue $\mathcal{A}$ must submit a decryption or encryption query with value $k$ such that $1=\mathcal{W E}$.iAtk $\left(\mathcal{W E}\right.$.pk, $\left.C_{\mathcal{W E}}, k\right)$. Since anything related to the value $b$ is encrypted with a value $k$ which is encrypted in $C_{\mathcal{W} \mathcal{E}, i}^{*}$ for $i=1$ to $n$, the only way for $\mathcal{A}$ to win is to issue an encryption or decryption query with $k$. Thus, the probability that $\mathcal{S}$ can break the one-way encryption $\mathcal{W E}$ is equivalent to the advantage of $\mathcal{A}$ in game PSI. 


\section{A.3. Proof of Theorem 3}

We sketch the proof of theorem 3 , which is similar to the proof of theorem 2.

Proof

The proof that our generic construction based on $\mathcal{B E}$ satisfies definitions 4,5,7 if the underlying MDVS $\mathcal{M S}$ satisfies the corresponding definitions is straightforward. We can easily construct a simulator $\mathcal{S}$ that acts as an attacker against $\mathcal{M S}$ if there exists an adversary $\mathcal{A}$ against the generic construction. $\mathcal{S}$ receives the system parameters of $\mathcal{M S}$, creates the public and secret keys of a oneway encryption $\mathcal{B E}$ for all users and gives them to $\mathcal{A}$. For any verification query submitted by $\mathcal{A}, \mathcal{S}$ can use the secret key of the $\mathcal{W E}$ to translate it to an appropriate query to the $\mathcal{M S}$. Finally, when $\mathcal{A}$ submits a forgery, $\mathcal{S}$ can decrypts it to produce the forgery of the underlying $\mathcal{M S}$.

We focus on the proof against privacy of signer's identity. Let $\mathcal{A}$ be an adversary with non-negligible advantage in game PSI. We show how to construct a simulator $\mathcal{S}$ that breaks the one-way security of the encryption $\mathcal{B E}$ in the ideal cipher model. $\mathcal{S}$ is given an instance of $\mathcal{B E}=\left(\right.$ param $_{\mathcal{B E}}, \mathcal{B E} \cdot$ pk $_{1}, \ldots, \mathcal{B E}$. pk $\left._{n}\right)$ and a challenge ciphertext $\left(C_{\mathcal{B E}}, \mathcal{Y}_{\mathcal{B E}}\right)$ where $\mathcal{Y}_{\mathcal{B E}}=$ $\left\{\mathcal{B E} \cdot \mathrm{pk}_{1}, \ldots, \mathcal{B E} \cdot \mathrm{pk}_{n}\right\}$ and its goal is to output the corresponding plaintext. $\mathcal{S}$ creates the parameter of $\mathcal{M S}$ honestly and is in possession of the keys for the two signers and the set of verifiers, say $\left(\mathcal{M S} . \mathrm{pk}_{i}, \mathcal{M S}\right.$.sk $\left.{ }_{i}\right)$ for verifier $V_{i} . \mathcal{S}$ sets the public keys of the verifiers as $\left(\mathcal{M S} . \mathrm{pk}_{i}, \mathcal{B E} . \mathrm{pk}_{i}\right)$ for verifier $V_{i} . \mathcal{S}$ chooses a symmetric cipher $\mathcal{S E}$ and a hash function $H . \mathcal{S E}$ is modelled as an idealized cipher.

Setup $\mathcal{S}$ gives $\left(\mathcal{M S}\right.$.param, param $\left.{ }_{\mathcal{B E}}, \mathcal{S E}, H\right)$ to $\mathcal{A}$ as param, together with the public keys of the signers and verifiers.

Query $\mathcal{S}$ uses the secret key of the signers to answer signature queries. Other types of queries are discussed below.

- For verification query of signature $(E, \mathcal{V}, C)$ on message $m, \mathcal{S}$ looks through the encryption query on $\mathcal{S E}$ with key $k$ that produces $E$. If the query is not found, return invalid. Otherwise, $\mathcal{S}$ checks if $1=\mathcal{B E}$. $\operatorname{Atk}\left(\mathcal{Y}_{\mathcal{B E}}, C, k\right)$. If not, $\mathcal{S}$ returns invalid. $\mathcal{S}$ obtains $\left(\sigma_{\mathcal{M S}}, \tau, \mathrm{pk}_{S}\right)$ from the encryption query of $\mathcal{S E}$ with output $E$. $\mathcal{S}$ returns invalid if $\tau \neq H\left(\mathrm{pk}_{S}, \mathrm{pk}_{V_{1}}, \ldots, \mathrm{pk}_{V_{n}}, C_{\mathcal{B E}}, m\right)$. Otherwise, $\mathcal{S}$ returns the verification results of $\sigma_{\mathcal{M S}}$ with secret key $\mathcal{M S}$.sk ${ }_{i}$.

- For each encryption or decryption query submitted by $\mathcal{A}$ with key $k, \mathcal{S}$ invokes $0 / 1 \leftarrow$ $\mathcal{B E}$.iAtk $\left(\mathcal{Y}_{\mathcal{B E}}, C_{\mathcal{W E}}, k\right)$. If the result is $1, \mathcal{S}$ returns $k$ and break the one-way security of $\mathcal{B E}$.

Challenge At some point $\mathcal{A}$ submits a message $m^{*}$. $\mathcal{S}$ flips a fair coin $b \in\{0,1\}$ and computes $\tau=H\left(\mathrm{pk}_{S_{b}}, \mathrm{pk}_{V_{1}}, \ldots, \mathrm{pk}_{V_{n}}, C_{\mathcal{B E}}, m\right)$. Invoke $\left(\sigma_{\mathcal{M S}}, \mathcal{V}_{\mathcal{M S}}\right) \leftarrow \operatorname{Sign}\left(\mathcal{M S} . \mathrm{sk}_{S_{b}}, \mathcal{V}_{\mathcal{M S}}, m^{*} \| \tau\right)$. Pick a random value $E^{*}$. Return $\left(E^{*}, \mathcal{V}, C_{\mathcal{B E}}\right)$ as the challenge signature. This implicitly sets $E^{*}=\mathcal{S E}$. Enc $_{k}\left(\sigma_{\mathcal{M S}} \| \tau|| \mathrm{pk}_{S_{b}}\right)$ where $k$ is the value encrypted in $C_{\mathcal{B E}}$.

Query Same as the previous query phase.

Output $\mathcal{A}$ outputs a guess bit $b^{\prime}$.

It remains to argue $\mathcal{A}$ must submit a decryption or encryption query with value $k$ such that $1=\mathcal{B E}$.iAtk $\left(\mathcal{Y}_{\mathcal{B E}}, C_{\mathcal{W E}}, k\right)$. Since anything related to the value $b$ is encrypted with a value $k$ which is encrypted in $\left(C_{\mathcal{B E}}, \mathcal{Y}_{\mathcal{B E}}\right)$, the only way for $\mathcal{A}$ to win is to issue an encryption or decryption query with $k$. Thus, the probability that $\mathcal{S}$ can break the one-way encryption $\mathcal{B E}$ is equivalent to the advantage of $\mathcal{A}$ in game PSI. 